Homology, Homotopy and Applications, vol.9(1), 2007, pp.221-256

\title{
THE FUNDAMENTAL WEIGHTED CATEGORY OF A WEIGHTED SPACE: FROM DIRECTED TO WEIGHTED ALGEBRAIC TOPOLOGY
}

\author{
MARCO GRANDIS
}

(communicated by George Janelidze)

\begin{abstract}
We want to investigate 'spaces' where paths have a 'weight', or 'cost', expressing length, duration, price, energy, etc. The weight function is not assumed to be invariant up to pathreversion. Thus, 'weighted algebraic topology' can be developed as an enriched version of directed algebraic topology, where illegal paths are penalised with an infinite cost, and the legal ones are measured. Its algebraic counterpart will be 'weighted algebraic structures', equipped with a sort of directed seminorm.

In the fundamental weighted category of a generalised metric space, introduced here, each homotopy class of paths has a weight (or seminorm), which is subadditive with respect to composition. We also study a more general setting, spaces with weighted paths, which has finer quotients and strong links with noncommutative geometry. Weighted homology of weighted cubical sets has already been developed in a previous work, with similar results.
\end{abstract}

\section{Introduction}

The recent domain of directed algebraic topology studies 'directed spaces' (preordered topological spaces, locally preordered spaces, cubical sets, etc.) with 'directed algebraic structures' produced by homotopy or homology functors: on the one hand the fundamental category (with its higher dimensional versions), on the other preordered homology groups. Its general aim is modelling non-reversible phenomena. See $[\mathbf{9}, \mathbf{1 0}, \mathbf{1 3}]$ and references therein.

We want to propose an enrichment of this subject, weighted algebraic topology, replacing the truth-valued approach of directed algebraic topology (where a path is legal or not) with a measure of costs, taking values in the interval $[0, \infty]$ of positive

Work supported by MIUR Research Projects.

Received April 24, 2006, revised July 24, 2006; published on February 12, 2007.

2000 Mathematics Subject Classification: 55Pxx, 54E35, 46L80.

Key words and phrases: homotopy theory, generalised metric space, directed algebraic topology, fundamental category, normed category, irrational rotation $\mathrm{C}^{*}$-algebra.

Copyright (C) 2007, International Press. Permission to copy for private use granted. 
real extended numbers. The general aim is now measuring the cost of (possibly non-reversible) phenomena.

Weighted algebraic topology will study 'weighted spaces', like (generalised) metric spaces, with 'weighted' algebraic structures, like the fundamental weighted (or normed) category, defined here, and the weighted homology groups already developed in [12] for weighted (or normed) cubical sets.

Lawvere's generalised metric spaces [18], endowed with a possibly non-symmetric distance taking values in $[0, \infty]$, are a basic setting where weighted algebraic topology can be developed (see Section 1). The present theory is essentially based on the standard generalised metric interval $\delta \mathbf{I}$, with distance $\delta(x, y)=y-x$, if $x \leqslant y$, and $\delta(x, y)=\infty$ otherwise; and the resulting cylinder functor $I(X)=X \otimes \delta \mathbf{I}$, where the tensor product has the $l_{1}$-type metric (Section 2$)$. We define the fundamental weighted category $\mathrm{w} \Pi_{1}(X)$ of a generalised metric spaces, and begin its study (Sections 3, 4).

As with various situations in homotopy theory, we have to work with elementary and extended homotopies, produced by 1-Lipschitz maps (i.e., weak contractions) and Lipschitz maps, respectively (see Section 2). The first are used to define the main homotopical constructs, namely cylinder, cone, suspension and - duallycocylinder, cocone, loop-object (in the pointed case); and then to obtain the (co-)fibration sequence of a map. But extended homotopies can be concatenated, and are essential to reach the higher order properties of such sequences. Also, in the fundamental weighted category itself, an arrow is a class of extended paths, up to extended homotopy with fixed endpoints.

(The reader can think of the following analogy: when studying homotopies of chain algebras, the multiplicative homotopies are used to define the main constructs, including the fibration sequence of a morphism, but such homotopies cannot be concatenated, and we need the 'extended' homotopies of the underlying chain complexes to prove the homotopy equivalence properties of the sequence; cf. [8].)

We also introduce, in Sections 5-6, the more flexible setting of $w$-spaces, or spaces with weighted paths, which - like weighted cubical sets in $[\mathbf{1 2}]$ - is able to express topological facts usually investigated with noncommutative geometry and missed by ordinary topology.

We only treat, in Section 7, an example, linked with the well-known irrational rotation $C^{*}$-algebras $A_{\vartheta}(\vartheta$ irrational) classified by Pimsner-Voiculescu [19] and Rieffel [20], also known as 'noncommutative tori'. The algebra $A_{\vartheta}$ is 'meant' to replace the topological quotient $\mathbf{R} / G_{\vartheta}$ of the euclidean line modulo the action of the dense additive group $G_{\vartheta}=\mathbf{Z}+\vartheta \mathbf{Z}$, which is trivial (i.e., has the coarse topology). Here, the 'metric' analogue $(\delta \mathbf{R}) / G_{\vartheta}$ is trivial as well, with the null distance. But we get an interesting quotient from the standard $w$-line $\mathrm{w} \mathbf{R}$, which assigns a finite weight $w(a)=a(1)-a(0)$ to each increasing path $a: \mathbf{I} \rightarrow \mathbf{R}$, and $w(a)=\infty$ otherwise (Subsection 5.4). Now, the w-space $W_{\vartheta}=(w \mathbf{R}) / G_{\vartheta}$ has a non-trivial fundamental weighted monoid (at any point), isomorphic to the additive monoid $G_{\vartheta}^{+}=G_{\vartheta} \cap \mathbf{R}^{+}$with the natural weight $w(x)=x$. We prove in Theorems $7.2,7.3$ that the irrational rotation $w$-space $W_{\vartheta}$ has the same classification up to isometric isomorphism (resp. Lipschitz isomorphism) as the $\mathrm{C}^{*}$-algebra $A_{\vartheta}$ up to isomorphism 
(resp. strong Morita equivalence).

\section{Generalised metric spaces}

We begin by recalling the structure of Lawvere's generalised metric spaces [18], which will be used as a first setting for weighted algebraic topology. Among the new points, notice the standard spaces of Subsection 1.5, the reflective symmetric distance (Subsection 1.6) and the associated symmetric topology (Subsection 1.7).

\subsection{Real weights}

The basic ingredient is the strict symmetric monoidal closed category of extended positive real numbers, introduced by Lawvere $[\mathbf{1 8}]$, which we write $\mathbf{w}^{+}$. It has objects $\lambda \in[0, \infty]$, morphisms $\lambda \geqslant \mu$, and tensor product $\lambda+\mu$ (with $\lambda+\infty=\infty$, for all $\lambda)$.

As a complete lattice, this category has all limits and colimits, reduced to products and sums

$$
\begin{aligned}
& \text { product: } \sup \lambda_{i}, \quad \text { terminal object: } 0, \\
& \text { sum: } \quad \inf \lambda_{i}, \quad \text { initial object: } \infty \text {. }
\end{aligned}
$$

The internal hom is given by truncated subtraction, written as a difference:

$$
\lambda+\mu \geqslant \nu \Leftrightarrow \lambda \geqslant \operatorname{hom}^{+}(\mu, \nu)=\nu-\mu, \quad(\nu-\mu=0 \vee(\nu-\mu)) .
$$

Let $\mathbf{v}$ denote the full subcategory of $\mathbf{w}^{+}$on the objects $0, \infty$; in this subcategory, the cartesian product $\lambda \vee \mu$ coincides with the tensor product $\lambda+\mu$. Thus, the covariant embedding of the boolean algebra $\mathbf{2}=(\{0,1\}, \leqslant)$ of truth-values (contravariant with respect to the natural orders)

$$
M: \mathbf{2} \rightarrow \mathbf{w}, \quad M(0)=\infty, \quad M(1)=0,
$$

is strict monoidal with respect to the cartesian product and the additive tensor product as well. Moreover, $M$ has left and right adjoint

$$
P \dashv M \dashv Q, \quad P(\lambda)=1 \Leftrightarrow \lambda<\infty, \quad Q(\lambda)=1 \Leftrightarrow \lambda=0 .
$$

A function $w: A \rightarrow[0, \infty]$ defined on a set equipped with a partial operation $a * b$, will be said to be (sub)additive if $w(a * b) \leqslant w(a)+w(b)$ whenever $a * b$ is defined; and strictly additive, or linear, if $w(a * b)=w(a)+w(b)$ (when this makes sense). The main property being the former, the prefix 'sub' will generally be omitted: for instance, an 'additively weighted' category has an 'additive' weight function on morphisms (Subsection 3.1), in the first sense.

Occasionally, we shall also use the same category $\mathbf{w}=([0, \infty], \geqslant)$ with the multiplicative structure $\mathbf{w}^{\bullet}$, where the tensor product is $\lambda$. $\mu$, and $\lambda . \infty=\infty$ for all $\lambda$ (cf. [14]). Also, here a multiplicative function is actually submultiplicative.

\subsection{Directed metrics}

Now, a generalised metric space $X$, in the sense of Lawvere [18], called here a directed metric space or $\delta$-metric space, is a set $X$ equipped with a $\delta$-metric 
$\delta: X \times X \rightarrow[0, \infty]$, satisfying the axioms

$$
\delta(x, x)=0, \quad \delta(x, y)+\delta(y, z) \geqslant \delta(x, z) .
$$

This corresponds to a category enriched over the symmetric monoidal closed category $\mathbf{w}^{+}$considered above, with $\delta(x, y)=X(x, y)$ the hom-object in $[0, \infty]$. (If the value $\infty$ is forbidden, $\delta$ is often called a quasi-pseudo-metric, cf. [15]; but including it has crucial advantages, e.g. with respect to limits and colimits.)

$\delta$ Mtr will denote the category of such $\delta$-metric spaces, with (weak) contractions, or 1-Lipschitz maps $f: X \rightarrow Y$, satisfying $\delta\left(x, x^{\prime}\right) \geqslant \delta\left(f(x), f\left(x^{\prime}\right)\right)$ for all $x, x^{\prime} \in$ $X$, also called $\delta$-maps. Isomorphisms in this category are isometric - and will be called isometric isomorphisms or 1-Lipschitz isomorphisms when needed. Limits and colimits exist and are calculated as in Set.

Products have the $l_{\infty}$-type $\delta$-metric (always defined because $\infty$ is included):

$$
\prod X_{i}: \quad \delta(\mathbf{x}, \mathbf{y})=\sup \delta_{i}\left(x_{i}, y_{i}\right) \quad\left(\mathbf{x}=\left(x_{i}\right), \mathbf{y}=\left(y_{i}\right)\right) .
$$

Equalisers have the restricted $\delta$-metric. Sums have the obvious $\delta$-metric (using $\infty$ also in the binary case):

$$
\sum X_{i}: \quad \delta((x, i),(y, i))=\delta_{i}(x, y) \quad \delta((x, i),(y, j))=\infty(i \neq j) .
$$

Coequalisers have the $\delta$-metric induced on the quotient:

$$
\begin{array}{ll}
X / R: \quad & \delta(\xi, \eta)=\inf _{\mathbf{x}}\left(\sum \delta\left(x_{2 j-1}, x_{2 j}\right)\right) \\
& \left(\mathbf{x}=\left(x_{1}, \ldots, x_{2 p}\right) ; x_{1} \in \xi ; x_{2 j} R x_{2 j+1} ; x_{2 p} \in \eta\right) .
\end{array}
$$

The term 'directed' (used here) refers to the non-symmetric character of $\delta$-metric spaces. And indeed such an object has a canonical preorder (to be used later, in Subsection 1.7)

$$
x \prec_{\infty} y \quad \text { if } \quad \delta(x, y)<\infty .
$$

More formally, a preordered set is the same as a $\delta$-metric space with a truthvalued metric $\delta: X \times X \rightarrow \mathbf{v}$, taking values in $\{0, \infty\}$; the canonical preorder (2) thus gives the left adjoint of the embedding of preordered sets into $\delta$-metric spaces (cf. (1)).

The reflected, or opposite, $\delta$-metric space $R(X)=X^{\mathrm{op}}$ has the opposite $\delta$-metric, $\delta^{\mathrm{op}}(x, y)=\delta(y, x)$. A symmetric $\delta$-metric, with $\delta=\delta^{\mathrm{op}}$, is the same as an écart in Bourbaki [3]. More generally, a $\delta$-metric space is reflexive, or self-dual, if it is isometrically isomorphic to its opposite (cf. Subsection 1.5). The notation $X \leqslant X^{\prime}$ means that these $\delta$-metric spaces have the same underlying set and $\delta_{X} \leqslant \delta_{X^{\prime}}$, or equivalently that the identity of the underlying set is a $\delta$-map $X^{\prime} \rightarrow X$.

\subsection{Lipschitz maps}

We will also use the wider category $\delta_{\infty}$ Mtr of $\delta$-metric spaces and all Lipschitz maps $f: X \rightarrow Y$, i.e. those mappings between the underlying sets for which the 
Lipschitz weight $\|f\|$ is finite:

$$
\|f\|=\min \left\{\lambda \in[0, \infty] \mid \text { for all } x, x^{\prime} \in X, \delta\left(f(x), f\left(x^{\prime}\right)\right) \leqslant \lambda . \delta\left(x, x^{\prime}\right)\right\},
$$

also called $\delta_{\infty}$-maps. A Lipschitz isomorphism will be an isomorphism of this category.

This category is finitely complete and cocomplete, with the same finite limits and colimits as $\delta \mathbf{M t r}$; but note that, now, the $\delta$-metric of a (co)limit is only determined up to Lipschitz-equivalence. Moreover, $\delta_{\infty} \mathbf{M t r}$ is multiplicatively weighted $[\mathbf{1 4}]$, with the Lipschitz weight: all identities have $\left\|1_{X}\right\| \leqslant 1$ and composition gives $\|g f\| \leqslant$ $\|f\| .\|g\|$. This also holds for $\delta$ Mtr, where $\|f\| \leqslant 1$.

If $X$ is a $\delta$-metric space and $\lambda \in[0, \infty$ [, we will write $\lambda X$ the same set equipped with the $\delta$-metric $\lambda . \delta_{X}$ (where it is assumed that $\lambda . \infty=\infty$ for all $\lambda$, cf. [14]). Thus, a $\delta_{\infty}$-map $f: X \rightarrow Y$ with $\|f\| \leqslant \lambda$ is the same as a $\delta$-map $\lambda X \rightarrow Y$. More generally, as in [18], one can define $\lambda X$ where $\lambda:[0, \infty] \rightarrow[0, \infty]$ is any increasing mapping with $\lambda(0)=0$ and $\lambda(\mu+\nu) \leqslant \lambda(\mu)+\lambda(\nu)$ (a lax monoidal functor $\mathbf{w}^{+} \rightarrow \mathbf{w}^{+}$). For instance, the square-root mapping gives the $\delta$-metric space $\sqrt{ } X$.

\subsection{Tensor product}

The category $\delta$ Mtr has a 'natural' symmetric monoidal closed structure (cf. [18], p. 153). The tensor product $X \otimes Y$ is the cartesian product of the underlying sets, with the $l_{1}$-type $\delta$-metric (instead of the $l_{\infty}$-type $\delta$-metric of the categorical product)

$$
\delta\left((x, y),\left(x^{\prime}, y^{\prime}\right)\right)=\delta\left(x, x^{\prime}\right)+\delta\left(y, y^{\prime}\right)
$$

It solves the usual universal problem, with respect to mappings which are 1-Lipschitz in each variable.

The exponential $Z^{Y}$ is the set of 1-Lipschitz maps $Y \rightarrow Z$ equipped with the $\delta$-metric of uniform convergence

$$
\begin{aligned}
& \delta(h, k)=\sup _{y} \delta_{Z}(h(y), k(y)) \\
& =\sup _{y y^{\prime}}\left(\delta_{Z}\left(h(y), k\left(y^{\prime}\right)\right)-\delta_{Y}\left(y, y^{\prime}\right)\right) \quad\left(\text { with } y, y^{\prime} \text { varying in } Y\right) \text {. }
\end{aligned}
$$

The proof of the adjunction is standard (and can be deduced from the proof of Theorem 5.1). The cartesian and tensor product are linked by the inequalities

$$
X \times Y \leqslant X \otimes Y \leqslant 2 .(X \times Y)
$$

In $\delta_{\infty}$ Mtr, these products are isomorphic and denote isomorphic functors (in two variables). But we shall keep distinguishing such objects (and functors); the notation $X \times Y$ (resp. $X \otimes Y$ ) still denotes the realisation of the cartesian product given by the $l_{\infty}$-type (resp. $l_{1}$-type) $\delta$-metric.

\subsection{Standard models}

The line $\mathbf{R}$ and the standard interval $\mathbf{I}$ will have the euclidean metric $|x-y|$. Then, $\mathbf{R}^{n}$ and $\mathbf{I}^{n}$ have the product metric, $\sup _{i}\left|x_{i}-y_{i}\right|$, while $\mathbf{R}^{\otimes n}$ and $\mathbf{I}^{\otimes n}$ have the tensor product metric, $\sum\left|x_{i}-y_{i}\right|$. 
But we are more interested in the following non-symmetric $\delta$-metrics. The standard $\delta$-line $\delta \mathbf{R}$ has the $\delta$-metric

$$
\delta(x, y)=y-x, \text { if } x \leqslant y, \quad \delta(x, y)=\infty, \text { otherwise; }
$$

its associated preorder is the natural order $x \leqslant y$ (cf. (2)).

The standard $\delta$-interval $\delta \mathbf{I}=\delta[0,1]$ has the subspace structure of the $\delta$-line. This also provides the cartesian powers $\delta \mathbf{R}^{n}, \delta \mathbf{I}^{n}$ and the tensor powers $\delta \mathbf{R}^{\otimes n}, \delta \mathbf{I}^{\otimes n}$. These $\delta$-metric spaces are not symmetric (for $n>0$ ), but reflexive; in particular, the canonical reflecting isomorphism

$$
r: \delta \mathbf{I} \rightarrow(\delta \mathbf{I})^{\mathrm{op}}, \quad t \mapsto 1-t,
$$

will play a role, in reflecting paths and homotopies (in the opposite space).

The standard $\delta$-circle $\delta \mathbf{S}^{1}$ will be the coequaliser in $\delta \mathbf{M t r}$ of the following two pairs of maps (equivalently)

$$
\begin{array}{ll}
\partial^{-}, \partial^{+}:\{*\} \rightrightarrows \delta \mathbf{I}, & \partial^{-}(*)=0, \quad \partial^{+}(*)=1, \\
\mathrm{id}, f: \delta \mathbf{R} \rightrightarrows \delta \mathbf{R}, & f(x)=x+1 .
\end{array}
$$

Thus, the 'standard realisation' of the first coequaliser is the quotient $(\delta \mathbf{I}) / \partial \mathbf{I}$, which identifies the endpoints; $\delta(x, y)$ takes values in $[0,1]$, and can be viewed as measuring the length of the 'counterclockwise arc' from $x$ to $y$, with respect to the whole circle. The structure $2 \pi . \delta \mathbf{S}^{1}$ is also of interest (now, arcs are measured with respect to the radius).

More generally, the $n$-dimensional $\delta$-sphere will be the quotient of the monoidal $\delta$-cube $\delta \mathbf{I}^{\otimes n}$ modulo its (ordinary) boundary $\partial \mathbf{I}^{n}$,

$$
\delta \mathbf{S}^{n}=\left(\delta \mathbf{I}^{n}\right) /\left(\partial \mathbf{I}^{n}\right) \quad(n>0),
$$

while $\delta \mathbf{S}^{0}=\{-1,1\}$ will be given the discrete $\delta$-metric, infinite out of the diagonal (so that every mapping from this space to any other be a contraction). All $\delta$-spheres are reflexive.

\subsection{The symmetric case}

The full subcategory Mtr of symmetric $\delta$-metric spaces (Subsection 1.2) has the same limits and colimits. Actually, it is reflective and coreflective in $\delta \mathbf{M t r}$.

The coreflector, right adjoint to the embedding, is the well-known symmetrising procedure $d\left(x, x^{\prime}\right)=\delta\left(x, x^{\prime}\right) \vee \delta\left(x^{\prime}, x\right)$, based on the least symmetric $\delta$-metric $d \geqslant \delta$. It will not be used here, since (for instance) it turns the $\delta$-metric of $\delta \mathbf{R}$ into the codiscrete $\delta$-metric - infinite out of the diagonal.

But we shall frequently use the reflector $!(X, \delta)=(X, ! \delta)$, based on the greatest symmetric $\delta$-metric $! \delta \leqslant \delta$

$$
\begin{aligned}
& ! \delta\left(x, x^{\prime}\right)=\inf _{\mathbf{x}}\left(\sum\left(\delta\left(x_{j-1}, x_{j}\right) \wedge \delta\left(x_{j}, x_{j-1}\right)\right)\right) \\
& \left(\mathbf{x}=\left(x_{0}, \ldots, x_{p}\right), x_{0}=x, x_{p}=x^{\prime}\right)
\end{aligned}
$$

which will be called the symmetrised $\delta$-metric (of $\delta$ ). The associated topology will be called the symmetric topology of the $\delta$-metric space $X$, and is the one we are interested in (see Subsection 1.7). 
This procedure turns the $\delta$-metric of $\delta \mathbf{R}$ into the euclidean metric. On $\delta \mathbf{R}^{n}$ the reflector gives a $\delta$-metric ! $\left(\delta \mathbf{R}^{n}\right)$ with $\epsilon$-disc as in the second figure below, the convex hull of $[-\epsilon, 0]^{n} \cup[0, \epsilon]^{n}$

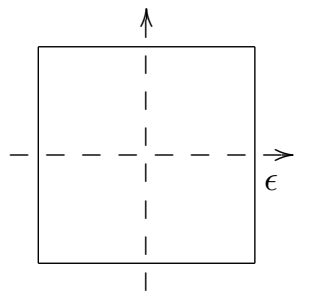

$(! \delta \mathbf{R})^{2}$

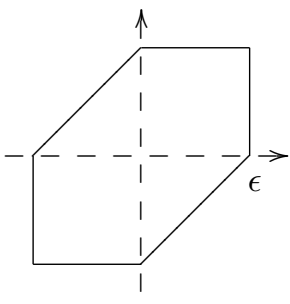

$!\left(\delta \mathbf{R}^{2}\right)$

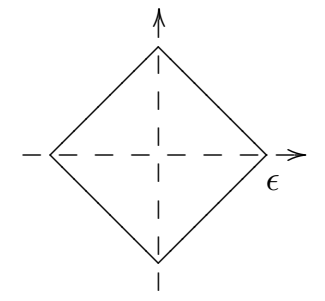

$!\left(\delta \mathbf{R}^{\otimes 2}\right)=(! \delta \mathbf{R})^{\otimes 2}$

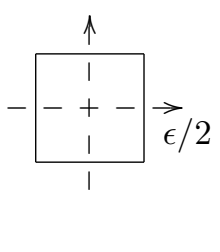

$2 .(! \delta \mathbf{R})^{2}$

while on the tensor powers $\delta \mathbf{R}^{\otimes n}$ it gives precisely the $l_{\infty}$-metric $(! \delta \mathbf{R})^{\otimes n}$, with $\epsilon$-disc as above, in the third figure. All these $\delta$-metrics are Lipschitz-equivalent, as follows from the figures above and from the following more general result.

Proposition 1.1 (Symmetrisation and products). Given a finite family of $\delta$-metric spaces $X_{1}, \ldots, X_{n}$ we have the following inequalities (or equalities) for the $\delta$-metrics obtained by symmetrisation, product and tensor product (on the cartesian product of the underlying sets $\left.\left|X_{1}\right| \times \ldots \times\left|X_{n}\right|\right)$

$$
\prod\left(! X_{i}\right) \leqslant !\left(\prod X_{i}\right) \leqslant !\left(\otimes X_{i}\right)=\bigotimes\left(! X_{i}\right) \leqslant n \cdot \prod\left(! X_{i}\right),
$$

so all these $\delta$-metrics are Lipschitz-equivalent and induce the same topology.

Proof. Recall the notation $\delta_{X}\left(x, x^{\prime}\right)=X\left(x, x^{\prime}\right)$, which comes from viewing a $\delta$ metric space as an enriched category (Subsection 1.2). The only non-standard point is the 'backward' inequality for the tensor product, proved in (c).

(a) First, to compare $\prod\left(! X_{i}\right)$ and !( $\left.\prod X_{i}\right)$, note that

$$
\prod\left(! X_{i}\right)(\mathbf{x}, \mathbf{y})=\sup \left(! X_{i}\right)\left(x_{i}, y_{i}\right) \leqslant \sup X_{i}\left(x_{i}, y_{i}\right)=\left(\prod X_{i}\right)(\mathbf{x}, \mathbf{y}) ;
$$

since $\prod\left(! X_{i}\right)$ is symmetric, it follows that $\prod\left(! X_{i}\right) \leqslant !\left(\prod X_{i}\right)$.

(b) The second inequality, ! $\left(\prod X_{i}\right) \leqslant !\left(\otimes X_{i}\right)$, follows from $\left(\prod X_{i}\right) \leqslant\left(\otimes X_{i}\right)$.

(c) We prove now that $!\left(\bigotimes X_{i}\right)=\bigotimes\left(! X_{i}\right)$. The $\delta$-metric of the latter is:

$$
\bigotimes\left(! X_{i}\right)(\mathbf{x}, \mathbf{y})=\sum_{i}\left(! X_{i}\right)\left(x_{i}, y_{i}\right) \leqslant \sum_{i} X_{i}\left(x_{i}, y_{i}\right)=\left(\bigotimes X_{i}\right)(\mathbf{x}, \mathbf{y}) .
$$

Since $\bigotimes\left(! X_{i}\right)$ is symmetric, we have $\bigotimes\left(! X_{i}\right) \leqslant !\left(\bigotimes X_{i}\right)$. The opposite inequality is more subtle: take a sequence of $n+1$ points $\mathbf{z}^{j}$, which vary from $\mathbf{x}$ to 
Homology, Homotopy and Applications, vol. 9(1), 2007

$\mathbf{y}$, by changing one coordinate at a time

$$
\mathbf{z}^{j}=\left(y_{1}, \ldots, y_{j}, x_{j+1}, \ldots, x_{n}\right), \quad \mathbf{z}^{0}=\mathbf{x}, \quad \mathbf{z}^{n}=\mathbf{y} \quad(j=0, \ldots, n),
$$

and apply the triangular inequality:

$$
!\left(X_{i}\right)(\mathbf{x}, \mathbf{y}) \leqslant \sum_{j} !\left(\bigotimes X_{i}\right)\left(\mathbf{z}^{j-1}, \mathbf{z}^{j}\right) .
$$

Now, $\mathbf{z}^{j-1}$ and $\mathbf{z}^{j}$ only differ at the $j$-th coordinate $\left(x_{j}\right.$ or $y_{j}$, respectively); restricting the domain of the 'inf' in the right term above to those sequences in $!\left(\otimes X_{i}\right)$ where only the $j$-th coordinate changes, we get the $\delta$-metric $! X_{j}$, and the inequality:

$$
\sum_{j} !\left(X_{i}\right)\left(\mathbf{z}^{j-1}, \mathbf{z}^{j}\right) \leqslant \sum_{j}\left(! X_{j}\right)\left(x_{j}, y_{j}\right)=\bigotimes\left(! X_{i}\right)(\mathbf{x}, \mathbf{y}) .
$$

(d) Finally, the last inequality in (6) is obvious.

Definition and Proposition 1.2 (The length of paths). Let $X$ be a $\delta$-metric space and $a: \mathbf{I} \rightarrow X$ a set-theoretical mapping. We define its span $s p(a)$ and its length $L(a)$ with the following functions, taking values in $[0, \infty]$ :

$$
\begin{array}{lr}
s p(a)=\sup _{\mathbf{t}} \delta\left(a\left(t_{0}\right), a\left(t_{1}\right)\right) & \left(\mathbf{t}=\left(t_{0}, t_{1}\right), 0 \leqslant t_{0}<t_{1} \leqslant 1\right), \\
L(a)=\sup _{\mathbf{t}} L_{\mathbf{t}}(a), & \left(\mathbf{t}=\left(t_{0}, \ldots, t_{p}\right), 0=t_{0}<t_{1}<\cdots<t_{p}=1\right) . \\
L_{\mathbf{t}}(a)=\sum \delta\left(a\left(t_{j-1}\right), a\left(t_{j}\right)\right)
\end{array}
$$

These functions satisfy the following properties, where $0_{x}$ is the constant path at (any) point $x, a+b$ denotes (any) path-concatenation of consecutive paths and $\|a\|$ is the Lipschitz weight (Subsection 1.3):

(a) $\operatorname{sp}\left(0_{x}\right)=L\left(0_{x}\right)=0$,

(b) $s p(a+b) \leqslant s p(a)+s p(b), \quad L(a+b)=L(a)+L(b)$,

(c) $s p(a \rho) \leqslant s p(a), L(a \rho) \leqslant L(a)$ (for every weakly increasing map $\rho: \mathbf{I} \rightarrow \mathbf{I}$ ),

(d) $\operatorname{sp}(a \rho)=\operatorname{sp}(a), \quad L(a \rho)=L(a) \quad$ (for every increasing homeomorphism $\rho: \mathbf{I} \rightarrow$ I),

(e) $s p(a, b)=s p(a)+s p(b), \quad L(a, b)=L(a)+L(b)$ (for all paths $(a, b): \mathbf{I} \rightarrow X \otimes$ $Y)$,

(f) $\operatorname{sp}(a) \leqslant L(a) \leqslant\|a\|$,

(g) $L$ is the least function on set-theoretical paths which is strictly additive for concatenation, invariant for reparametrisation on increasing homeomorphisms $\mathbf{I} \rightarrow \mathbf{I}$, and satisfies $L \geqslant s p$,

(h) $s p(f \circ a) \leqslant\|f\| . s p(a), L(f \circ a) \leqslant\|f\| . L(a) \quad$ (for all $\delta_{\infty}$-maps $f: X \rightarrow Y$ ),

(i) for $a: \mathbf{I} \rightarrow \delta \mathbf{R}^{\otimes n}$, we get $L(a)=\operatorname{sp}(a)=\sum_{i}\left(a_{i}(1)-a_{i}(0)\right)$ if a is (weakly) increasing, and $L(a)=\infty$ otherwise.

Finally, note that the length $L(a)$ can be finite even when a is not Lipschitz: $\|a\|=\infty$. 
Proof. The properties of the span being obvious, we only verify the ones of the length. It will be useful to note that, if the partition $\mathbf{t}^{\prime}$ is finer than $\mathbf{t}$, then $L_{\mathbf{t}}(a) \leqslant$ $L_{\mathbf{t}^{\prime}}(a)$, because of the triangular property of $\delta$-metrics.

Point (a) is obvious. For (b), the inequality $L(a+b) \leqslant L(a)+L(b)$ follows easily: given a partition $\mathbf{t}$ for $c=a+b$, call $\mathbf{t}^{\prime}$ its refinement by introducing the point $t=\frac{1}{2}$ (if missing); thus $L_{\mathbf{t}}(c) \leqslant L_{\mathbf{t}^{\prime}}(c)$ and the latter term can be split into two summands $\leqslant L(a)+L(b)$. For the other inequality, it is sufficient to note that a partition for $a$ and one for $b$ determine a partition of $[0,2]$, which can be scaled down to the standard interval.

Point (c) is obvious, since $L(a \rho)$ is computed on the partitions of $\rho[0,1] \subset[0,1]$; (d) is a consequence. For (e), the inequality $L(a, b) \leqslant L(a)+L(b)$ is obvious, and the other follows again from the first remark: given a partition for $a$ and one for $b$, by using a common refinement for both we get higher values.

Finally, (f)-(h) are plain; (i) is obvious for $n=1$, and follows from (e) for higher $n$. For the last remark, taking $X=\delta \mathbf{R}$, the square-root map $f: \mathbf{I} \rightarrow \mathbf{R}$ is not Lipschitz but has a finite length, as any increasing path: $L(f)=f(1)-f(0)=1$.

\subsection{The associated topology and direction}

A $\delta$-metric space $X$ has an obvious directed topology, generated by the open discs $\left\{x \mid \delta\left(x_{0}, x\right)<\epsilon\right\}$. We will not use this construct.

Rather, we shall use the symmetric topology, determined as above by the symmetric $\delta$-metric $! \delta$ defined in (5), and we will keep a trace of the 'directed' information of the original $\delta$, in various ways, more or less effective, based on the settings for directed homotopy used in $[\mathbf{9}]$.

The simplest, if rather poor way, is by the associated preorder $x \prec_{\infty} y$, defined by $\delta(x, y)<\infty(2)$. We thus have a forgetful functor with values in the category pTop of preordered spaces and continuous preorder-preserving mappings

$$
\mathbf{p}: \delta \text { Mtr } \rightarrow \text { pTop }, \quad\left(\delta_{\infty} \text { Mtr } \rightarrow \text { pTop }\right),
$$

which preserves finite products (since the symmetrising functor !: $\delta \mathbf{M t r} \rightarrow \mathbf{M t r}$ preserves finite products up to Lipschitz equivalence (1.1), and $\delta(x, y)<\infty$ if and only if this holds on all components).

Thus, $\mathbf{p}\left(\delta \mathbf{R}^{n}\right)=\mathbf{p}\left(\delta \mathbf{R}^{\otimes n}\right)=\uparrow \mathbf{R}^{n}$, the euclidean $n$-dimensional space with the product order. Similarly, $\mathbf{p}\left(\delta \mathbf{I}^{n}\right)=\mathbf{p}\left(\delta \mathbf{I}^{\otimes n}\right)=\uparrow \mathbf{I}^{n}$. But a preorder is a poor way of describing direction, which does not allow for non-reversible loops. Thus, $\mathbf{p}\left(\delta \mathbf{S}^{1}\right)$ gets the chaotic preorder and misses any information on direction.

A more accurate way of keeping the 'directed' information of the original $\delta$ is using $d$-spaces, i.e. spaces with distinguished paths $[\mathbf{9}]$. We have thus a forgetful functor

$$
\mathbf{d}: \delta \mathbf{M t r} \rightarrow \mathrm{d} \text { Top, } \quad\left(\delta_{\infty} \mathbf{M t r} \rightarrow \mathrm{d} \mathbf{T o p}\right),
$$

which equips a $\delta$-metric space $X$ with the associated symmetric topology and the d-structure where a (continuous) path $a: \mathbf{I} \rightarrow X$ is distinguished if and only if it is L-feasible, i.e. it has a finite length $L(a)(7)$. The axioms of d-spaces are satisfied (by Definition and Proposition 1.2): distinguished paths contain all the constant ones, are closed under concatenation and partial reparametrisation by weakly 
increasing maps $\mathbf{I} \rightarrow \mathbf{I}$. And, of course, a Lipschitz map $f: X \rightarrow Y$ of $\delta$-metric spaces preserves feasible paths. It will be important to note that this functor takes the tensor (or cartesian) product in $\delta_{\infty} \mathbf{M t r}$ (or $\delta \mathbf{M t r}$ ) to the cartesian product of d-spaces (where a path is distinguished if and only if its two components are).

Now, in $\mathrm{d} \mathbf{T o p}, \mathbf{d}\left(\delta \mathbf{S}^{1}\right)=\uparrow \mathbf{S}^{1}=\uparrow \mathbf{I} / \partial \mathbf{I}$ is the standard directed circle [9], where path are only allowed to turn in a given direction. But the functor $\mathbf{d}$ need not preserve quotients: for instance, $\mathbf{d}(\delta \mathbf{R})=\uparrow \mathbf{R}$ is the standard directed line, with the increasing paths as distinguished ones; the quotient d-space $\uparrow \mathbf{R} / G_{\vartheta}$, modulo the action of the dense subgroup $G_{\vartheta}=\mathbf{Z}+\vartheta \mathbf{Z}$ (for an irrational number $\vartheta$ ) is a non-trivial object, with the homology of the 2-dimensional torus (cf. [12]), while $(\delta \mathbf{R}) / G_{\vartheta}$ has a trivial $\delta$-metric, always zero. A finer notion of 'weighted space', studied in Sections 5-7, will be able to express such phenomena within weighted algebraic topology (not just the directed one).

Note that the forgetful functor $\mathrm{d}$ Top $\rightarrow$ pTop provided by the path-preorder $x \preceq x^{\prime}$ (there is a distinguished path from $x$ to $x^{\prime}$ ), applied to a $\mathrm{d}$-space of type $\mathbf{d} X$, gives a finer preorder than $\mathbf{p} X$, generally more interesting than the latter (two points at a finite distance may be disconnected, or not linked by a feasible path.)

One could also try an intermediate way of codifying direction, by some notion of local preorder, as in Krishnan [16]. For instance, one could use a family of preorders, indexed on the open subsets $U$ of $X$ (always with respect to the symmetric topology)

$$
x \prec_{U} x^{\prime} \quad \text { if } \quad\left(x \preceq x^{\prime} \text { in the path-preorder of } U \text {, for the induced } \delta\right. \text {-metric), }
$$

so that if $U \subset V, x \prec_{U} x^{\prime}$ implies $x \prec_{V} x^{\prime}$. Thus, in $\delta \mathbf{S}^{1}$, every proper open arc $U$ gets a total order (Lipschitz isomorphic to an open interval).

\section{Elementary and extended homotopies}

The standard $\delta$-interval $\delta \mathbf{I}$ generates a cylinder endofunctor, which yields elementary homotopies in $\delta \mathbf{M t r}$, and extended homotopies in $\delta_{\infty} \mathbf{M t r}$. We need both, but only the latter can be concatenated. The letter $\alpha$ denotes an element of the set $\{0,1\}$, written,-+ in superscripts.

\subsection{Elementary and extended paths}

Let $X$ be a $\delta$-metric space. An elementary path (resp. an extended path, or Lipschitz path) in $X$ will be a 1-Lipschitz (resp. a Lipschitz) map $a: \delta \mathbf{I} \rightarrow X$.

Thus, a set-theoretical mapping $a: \mathbf{I} \rightarrow X$ is an elementary path if and only if $\|a\| \leqslant 1$, for the Lipschitz weight (3)

$$
\|a\|=\min \left\{\lambda \in[0, \infty] \mid \text { for all } t \leqslant t^{\prime} \text { in }[0,1], \delta\left(a(t), a\left(t^{\prime}\right)\right) \leqslant \lambda .\left(t^{\prime}-t\right)\right\},
$$

and is an extended path if and only if $\|a\|<\infty$. Elementary paths cannot be concatenated, because - loosely speaking - this procedure doubles the velocity, whose least upper bound is the Lipschitz weight. Recall that the (finite) length $L(a) \leqslant\|a\|$ has been defined in Definition and Proposition 1.2. 
Homology, Homotopy and Applications, vol. 9(1), 2007

The reflected (elementary or extended) path is obtained in the obvious way

$$
a^{\mathrm{op}}=a r: \delta \mathbf{I} \rightarrow X^{\mathrm{op}}, \quad r(t)=1-t .
$$

A reversible extended path is a mapping $a: \mathbf{I} \rightarrow X$ such that both $a$ and $a^{\text {op }}$ are extended paths $\delta \mathbf{I} \rightarrow X$. This corresponds to a Lipschitz map $a: ! \delta \mathbf{I} \rightarrow X$, with respect to the ordinary metric $\left|t-t^{\prime}\right|$ of the euclidean interval.

\subsection{Concatenation}

Given two consecutive Lipschitz paths $a, b: \delta \mathbf{I} \rightarrow X$, with $a(1)=b(0)$, the usual construction gives a concatenated path

$$
a+b: \delta \mathbf{I} \rightarrow X, \quad \quad\|a+b\| \leqslant 2 .(\|a\| \vee\|b\|),
$$

(which need not be elementary when $a$ and $b$ are). Let us review this fact in a more formal way. In the category $\delta \mathbf{M t r}$, pasting two copies of the standard $\delta$-interval, one after the other, can be realised as $\delta[0,2] \subset \delta \mathbf{R}$, or (isometrically) as $2 . \delta \mathbf{I}$ (with the double $\delta$-metric)

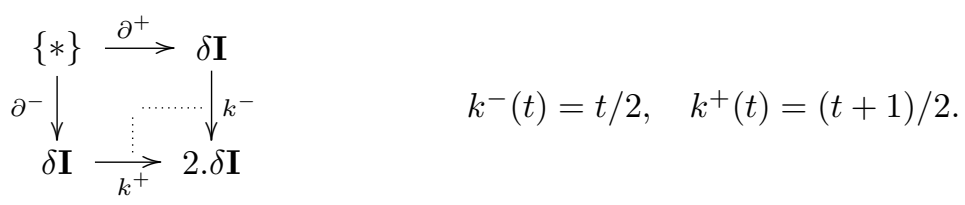

Of course, this is of no help to concatenate elementary paths. Moving to the category $\delta_{\infty} \mathbf{M t r}$, this pushout can be realised as the Lipschitz-isomorphic object $\delta \mathbf{I}$. This yields the standard concatenation pushout (the left diagram below, which actually lives in $\delta$ Mtr but is not a pushout there)
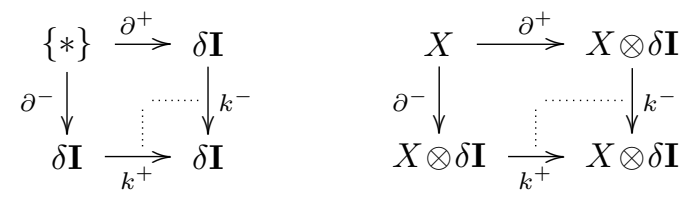

$$
\begin{aligned}
k^{-}(x, t) & =(x, t / 2) \\
k^{+}(x, t) & =(x,(t+1) / 2) .
\end{aligned}
$$

This pushout is preserved by any functor $X \otimes-$ (yielding the right hand pushout above), or by $X \times-$. In fact, $X \otimes-: \delta \mathbf{M t r} \rightarrow \delta \mathbf{M t r}$ preserves the pushout (10), as a left adjoint; and the embedding $\delta \mathbf{M t r} \rightarrow \delta_{\infty}$ Mtr preserves finite colimits (Subsection 1.3).

\subsection{The elementary cylinder}

The $\delta$-interval $\delta \mathbf{I}$ is an internal lattice in $(\delta \mathbf{M t r}, \otimes)$; its structure consists of two faces $\left(\partial^{-}, \partial^{+}\right)$, a degeneracy $(e)$, two connections or main operations $\left(g^{-}, g^{+}\right)$and an interchange $(s)$

$$
\begin{aligned}
& \{*\} \stackrel{\partial^{\alpha}}{\underset{\kappa_{e}}{\gtrless}} \delta \mathbf{I} \stackrel{g^{\alpha}}{\longleftarrow} \delta \mathbf{I}^{\otimes 2} \quad s: \delta \mathbf{I}^{\otimes 2} \rightarrow \delta \mathbf{I}^{\otimes 2} \quad(\alpha= \pm) \\
& \partial^{-}(*)=0, \quad \partial^{+}(*)=1, \\
& g^{-}\left(t, t^{\prime}\right)=t \vee t^{\prime}, \quad g^{+}\left(t, t^{\prime}\right)=t \wedge t^{\prime}, \quad s\left(t, t^{\prime}\right)=\left(t^{\prime}, t\right) \text {. }
\end{aligned}
$$


As a consequence, the elementary cylinder endofunctor

$$
I: \delta \mathbf{M t r} \rightarrow \delta \mathbf{M t r}, \quad I(-)=-\otimes \delta \mathbf{I},
$$

has natural transformations, which will be denoted by the same symbols and names

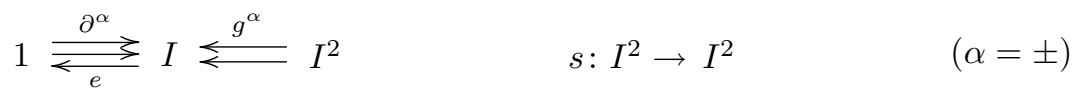

The functor $I$, with these transformations, satisfies the axioms of a cubical monad with interchange $[\mathbf{7}, \mathbf{8}]$ :

$$
\begin{array}{llr}
e \partial^{\alpha}=1, & e g^{\alpha}=e \cdot I e(=e . e I) & (\text { degeneracy axiom }), \\
g^{\alpha} \cdot I g^{\alpha}=g^{\alpha} \cdot g^{\alpha} I, & g^{\alpha} \cdot I \partial \alpha=1=g^{\alpha} \cdot \partial^{\alpha} I & (\text { associativity, unit), } \\
g^{\beta} \cdot I \partial^{\alpha}=\partial^{\alpha} e=g^{\beta} \cdot \partial^{\alpha} I & & (\text { absorbency, for } \alpha \neq \beta), \\
\text { s.s }=1, \quad \text { Ie.s }=e I, & \text { s.I } \partial^{\alpha}=\partial^{\alpha} I, \quad g^{\alpha} . s=g^{\alpha} & \text { (interchange) } .
\end{array}
$$

The cylinder $I$ has a generalised reversion, via the reflection of $\delta$-metric spaces (as always happens in directed algebraic topology, e.g. for d-spaces [9] and for differential graded algebras $[\mathbf{7}]$ )

$$
\begin{array}{lll}
r X=X \otimes r: I R X \rightarrow R I X, & (x, t) \mapsto(x, 1-t), & \\
R r R . r=\mathrm{id}, & \text { Re.r }=e R, & \\
r . \partial^{-} R=R \partial^{+}, & r . g^{-} R=R g^{+} . r_{2}, \quad \text { Rs. } r_{2}=r_{2} . s R .
\end{array}
$$

where $r_{2}=r I$.Ir $:\left(I^{2} R \rightarrow I R I \rightarrow R I^{2}\right)$ is the reversion of the double cylinder.

Within $\delta \mathbf{M t r}$, the cylinder endofunctor $I=-\otimes \delta \mathbf{I}$ has a right adjoint, the elementary-path functor, or elementary cocylinder

$$
P: \delta \mathbf{M t r} \rightarrow \delta \mathbf{M t r}, \quad P(Y)=Y^{\delta \mathbf{I}} .
$$

The $\delta$-metric space $Y^{\delta \mathbf{I}}$ is the set of elementary paths $\delta \mathbf{M t r}(\delta \mathbf{I}, Y)$ with the $\delta$ metric of uniform convergence (4). The lattice structure of $\delta \mathbf{I}$ in dTop producescontravariantly - a dual structure on $P$ (a cubical comonad with interchange $[\mathbf{7}, \mathbf{8}]$ ).

\subsection{Homotopies}

An elementary homotopy $\varphi: f \rightarrow g: X \rightarrow Y$ is defined as a $\delta$-map $\varphi: I X=X \otimes$ $\delta \mathbf{I} \rightarrow Y$ whose two faces are $f$ and $g$, respectively: $\partial^{-}(\varphi)=\varphi \circ \partial^{-}=f, \partial^{+}(\varphi)=$ $\varphi \circ \partial^{+}=g$. In particular, an elementary path is a homotopy between two points, $a: x \rightarrow x^{\prime}:\{*\} \rightarrow X$.

More generally, an extended homotopy, or Lipschitz homotopy, is a Lipschitz map $\varphi: X \times \delta \mathbf{I} \rightarrow Y$; and an extended path is an extended homotopy between two points, $a: x \rightarrow x^{\prime}:\{*\} \rightarrow X$. (Note that a Lipschitz map defined on the singleton is always a $\delta$-map.) An extended homotopy has a Lipschitz weight $\|\varphi\|$, which is $\leqslant 1$ if and only if $\varphi$ is elementary.

In both cases, the main operations produced by the cylinder functor (for $\varphi: f \rightarrow$ $\left.g: X \rightarrow Y ; u: X^{\prime} \rightarrow X ; v: Y \rightarrow Y^{\prime} ; \psi: g \rightarrow h: X \rightarrow Y\right)$ are:

(a) whisker composition of (elementary or extended) maps and homotopies

$$
v \circ \varphi \circ u: v f u \rightarrow v g u \quad\left(v \circ \varphi \circ u=v \cdot \varphi \cdot I u: I X^{\prime} \rightarrow Y^{\prime}\right),
$$


(b) trivial homotopies:

$$
0_{f}: f \rightarrow f \quad\left(0_{f}=f e: I X \rightarrow Y\right),
$$

and satisfy obvious axioms for units and associativity:

$$
1 \circ \varphi \circ 1=\varphi, \quad v \circ 0_{f} \circ u=0_{v f u}, \quad\left(v^{\prime} v\right) \circ \varphi \circ\left(u u^{\prime}\right)=v^{\prime} \circ(v \circ \varphi \circ u) \circ u^{\prime} .
$$

This 2-dimensional structure, weaker than a bicategory or a sesquicategory (there is no vertical composition of 2-cells) is called an $h$-category in [8] (it is a category enriched over reflexive graphs, with a suitable monoidal structure).

More precisely, we will write:

(i) $\delta_{1} \mathrm{Mtr}$, the h-category of $\delta$-metric spaces, with weak contractions and elementary homotopies,

(ii) $\delta_{\infty} \mathbf{M t r}$, the h-category of $\delta$-metric spaces, with Lipschitz maps and Lipschitz homotopies,

(iii) $\delta$ Mtr, the intermediate h-category of weak contractions and Lipschitz homotopies between them.

Actually, $\delta_{1}$ Mtr is a bicomplete IP-homotopical category [7]; it has adjoint functors $I \dashv P$, with the required structure (a cubical monad and a cubical comonad, respectively), colimits (preserved by the cylinder) and limits (preserved by the cocylinder). Therefore, all results of $[\mathbf{7}]$ for such a structure apply (as for cochain algebras).

Moreover, in $\delta_{\infty} \mathbf{M t r}$ (and $\delta \mathbf{M t r}$ ) consecutive homotopies can be pasted via the concatenation pushout of the cylinder functor (the right-hand diagram in (11)). The concatenation $\varphi+\psi$ of two consecutive homotopies $\left(\partial^{+} \varphi=\partial^{-} \psi\right)$ is thus computed as usual:

$$
(\varphi+\psi)(x, t)=\varphi(x, 2 t) \text { for } 0 \leqslant t \leqslant \frac{1}{2}, \quad=\psi(x, 2 t-1) \text { for } \frac{1}{2} \leqslant t \leqslant 1 .
$$

Reflected homotopies (elementary or extended) live in the opposite 'spaces' (as for paths, in (9))

$$
\varphi^{\mathrm{op}}: R g \rightarrow R f: R X \rightarrow R Y, \quad \varphi^{\mathrm{op}}=R \varphi \cdot r X=(I(R X) \rightarrow R(I X) \rightarrow R Y) .
$$

As always in directed algebraic topology, homotopy equivalence is a complex notion, which has to be considered not only for 'spaces' but also for their algebraic counterpart-weighted categories. This will be briefly considered in Section 5. A more complete study for d-spaces can be found in $[\mathbf{9}]$.

\subsection{Double homotopies and 2-homotopies}

Extended homotopies in dimension 2, based on the second order cylinder $I^{2} X=$ $X \otimes \delta \mathbf{I}^{2}$, can now be treated in the same way as for d-spaces, in [9]. Roughly speaking, they behave as in Top, as long as we work on the standard square $[0,1]^{2}$ with increasing Lipschitz maps. We will only sketch the main points; the interested reader can see $[\mathbf{9}], 2.5-2.6$.

An extended double homotopy is a map $\Phi: X \otimes \delta \mathbf{I}^{2}=I^{2} X \rightarrow Y$; it has four faces $\partial_{1}^{\alpha}(\Phi)$, with $\alpha= \pm$ and $i=1,2$. The concatenation of extended double homotopies 
in direction 1 or 2 is defined as usual (under the obvious boundary conditions) and satisfies a strict middle-four interchange property.

An extended 2-homotopy $\Phi: \varphi \rightarrow \psi: f \rightarrow g: X \rightarrow Y$ is a double homotopy whose faces $\partial_{1}^{\alpha}$ are degenerate, while the faces $\partial_{2}^{\alpha}$ are $\varphi, \psi$ (the other choice is equivalent, by interchange). Such particular double homotopies are closed under concatenations in each direction (also because $0_{f}+0_{f}=0_{f}$ ). The preorder $\varphi \preceq_{2} \psi$ (i.e., there is a 2-homotopy $\varphi \rightarrow \psi$ ) spans an equivalence relation $\simeq_{2}$.

The following constructions of 2-dimensional homotopies are crucial tools.

(a) Two 'horizontally' consecutive extended homotopies

$$
\varphi: f^{-} \rightarrow f^{+}: X \rightarrow Y, \quad \psi: g^{-} \rightarrow g^{+}: Y \rightarrow Z
$$

can be composed, to form an extended double homotopy $\Phi=\psi \circ \varphi$ (which is elementary if $\varphi$ and $\psi$ are)

$$
\begin{array}{cc}
g^{-} f^{-} \stackrel{g^{-} \varphi}{\longrightarrow} g^{-} f^{+} & \Phi=\psi \circ \varphi=\psi \cdot(\varphi \otimes \delta \mathbf{I}): X \otimes \delta \mathbf{I}^{2} \rightarrow Z, \\
\psi^{-} f_{\psi} \rightarrow \varphi \circ f^{+} & \partial_{1}^{\alpha}(\Phi)=\psi \cdot\left(\varphi \partial^{\alpha} \otimes \delta \mathbf{I}\right)=\psi \circ f^{\alpha}, \\
g^{+} f^{-} \underset{g^{+} \varphi}{\longrightarrow} g^{+} f^{+} & \partial_{2}^{\alpha}(\Phi)=\psi \cdot\left(\varphi \otimes \partial^{\alpha}\right)=g^{\alpha} \circ \varphi .
\end{array}
$$

(Together with the whisker composition in Subsection 2.4, this is a particular instance of the cubical enrichment produced by the cylinder functor: composing a $p$-uple homotopy $\Phi: I^{p} X \rightarrow Y$ with a $q$-uple one $\Psi: I^{q} Y \rightarrow Z$ gives a $(p+q)$-uple homotopy $\left.\Psi \circ \Phi=\Psi . I^{q} \Phi.\right)$

(b) Acceleration. For every extended homotopy $\varphi: f \rightarrow g$, there are acceleration extended 2-homotopies (cf. [9], 2.6.4)

$$
\Theta^{\prime}: 0_{f}+\varphi \rightarrow \varphi, \quad \Theta^{\prime \prime}: \varphi \rightarrow \varphi+0_{g},
$$

(but not the other way round: slowing down conflicts with direction).

(c) Folding. A double extended homotopy $\Phi: A \otimes \delta \mathbf{I}^{2} \rightarrow X$ with faces $\varphi, \psi, \sigma, \tau$ (as below) produces a 2-homotopy $\Psi$, by pasting $\Phi$ with two double homotopies produced by the connections $g^{ \pm}$(Subsection 2.3), denoted with $\sharp$

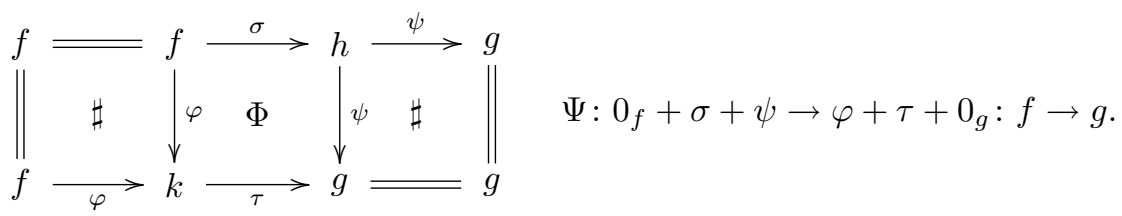

Together with accelerations, this 2-homotopy $\Psi$ shows that the faces of $\Phi$ satisfy $\sigma+\psi \simeq_{2} \varphi+\tau$.

\subsection{Homotopy constructs}

We have now the tools to develop the homotopy constructs of [10] for $\delta$-metric spaces: (a) homotopy pushouts and pullbacks; (b) mapping cones, suspension and 
cofibration sequences; (c) homotopy fibres, loop-objects and fibration sequences (in the pointed case).

The higher properties of this machinery then need concatenation, and can only be developed in $\delta_{\infty}$ Mtr. We will not write down these computations here.

\section{The fundamental weighted category}

The fundamental category of a $\delta$-metric space is defined. Non-obvious computations are based on a van Kampen-type theorem (Theorem 3.2) similar to R. Brown's version for the fundamental groupoid of a space [4] or to our version for a space with distinguished paths $[\mathbf{9}]$.

\subsection{Weighted categories}

An additively weighted category is a category $X$ where every morphism $a$ is equipped with a weight, or cost, $w(a) \in[0, \infty]$, so that two obvious axioms are satisfied, for identities and composition (written in additive notation):

$$
\begin{array}{ll}
\left(\mathrm{w}^{+} \text {cat. }\right) & w\left(0_{x}\right)=0, \\
\left(\mathrm{w}^{+} \text {cat. } 1\right) & w(a+b) \leqslant w(a)+w(b), \quad \text { for all pairs of consecutive arrows } a, b .
\end{array}
$$

This is called a normed category in $[\mathbf{1 8}, \mathbf{2}]$; see the Introduction and $[\mathbf{1 4}]$ for the present terminology on the additive and multiplicative variants. We will omit the term 'additive' when there is no ambiguity; we also speak of a $w^{+}$-category for short. The weight is said to be linear, or strictly additive, if $w(a+b)=w(a)+w(b)$ for all composites.

A $w^{+}$-functor $f: X \rightarrow Y$, or 1-Lipschitz functor, is a functor between such categories which satisfies the condition $w(f(a)) \leqslant w(a)$, for all morphisms $a$ of $X$. A $w^{+}$-transformation $\varphi: f \rightarrow g$ is a natural transformation between w-functors. All this forms the 2 -category $\mathrm{w}^{+}$Cat of $\left(\right.$small) $\mathrm{w}^{+}$-categories, also written ${ }^{+}$Cat. (Here we do not use the multiplicative analogue, for which see [14]).

Weighted categories can be viewed as categories enriched over the symmetric monoidal closed category $\mathrm{w}^{+}$Set of weighted sets (i.e., sets equipped with a mapping $w: X \rightarrow \mathbf{w}^{+}$, and maps $f: X \rightarrow Y$ such that $w(f(x)) \leqslant w(x)$, for all $\left.x \in X\right)$, with an 'additive' tensor product $[\mathbf{2}, \mathbf{1 4}]$.

The opposite weighted category $X^{\mathrm{op}}$ is the opposite category with the 'same' weight.

Also here (as for $\delta$-metric spaces, in Subsection 1.3) we need the wider category $\mathrm{w}_{\infty}$ Cat of weighted categories and Lipschitz functors $f: X \rightarrow Y$, or $\mathrm{w}_{\infty}$-functors, i.e. the functors between weighted categories having a finite Lipschitz weight

$$
\|f\|=\min \left\{\lambda \in[0, \infty] \mid \text { for all } a \in \operatorname{Mor}(X), w_{Y}(f(a)) \leqslant \lambda . w_{X}(a)\right\} .
$$

With this weight, the category $\mathrm{w}_{\infty}$ Cat is multiplicatively weighted (cf. Subsection 1.3). This also holds for wCat, which is the wide subcategory of the functors $f$ such that $\|f\| \leqslant 1$. 
Proposition 3.1 (The monoidal closed structure). The category wCat has a symmetric monoidal closed structure, with tensor product $X \otimes Y$ consisting of the cartesian product of the underlying categories with the $l_{1}$-type weight on a map $(a, b)$ : $(x, y) \rightarrow\left(x^{\prime}, y^{\prime}\right):$

$$
w_{\otimes}(a, b)=w(a)+w(b) .
$$

The internal hom $Z^{Y}$ is the category of 1-Lipschitz functors $h: Y \rightarrow Z$ and all natural transformations $\varphi: h \rightarrow k$ between such functors, with the (plainly subadditive) weight:

$$
W(\varphi)=\sup _{y} w_{Z}(\varphi(y)), \quad(y \in \mathrm{Ob} Y) .
$$

Proof. First a 1-Lipschitz functor $f: X \otimes Y \rightarrow Z$ defines a functor $g: X \rightarrow Z^{Y}$, sending an object $x$ to the 1-Lipschitz functor

$$
g(x)=f(x,-): Y \rightarrow Z, \quad w_{Z}(g(x)(b))=w_{Z}\left(f\left(0_{x}, b\right)\right) \leqslant w_{\otimes}\left(0_{x}, b\right)=w(b),
$$

and the $X$-morphism $a: x \rightarrow x^{\prime}$ to the natural transformation $g(a)=f(a,-): g(x)$ $\rightarrow g\left(x^{\prime}\right)$. The functor $g$ itself is a contraction:

$$
W(g(a))=\sup _{y} w_{Z}(g(a)(y))=\sup _{y} w_{Z}\left(f\left(a, 0_{y}\right)\right) \leqslant \sup _{y} w_{\otimes}\left(a, 0_{y}\right)=w(a) .
$$

Conversely, given a 1-Lipschitz functor $g: X \rightarrow Z^{Y}$, we define the functor $f: X \otimes$ $Y \rightarrow Z$ in the usual, obvious way, and verify that it is 1-Lipschitz, on a map $(a, b):(x, y) \rightarrow\left(x^{\prime}, y^{\prime}\right)$

$$
w_{Z}(f(a, b))=w_{Z}(g(a)(y)+g(x)(b)) \leqslant w_{Z}(g(a)(y))+w_{Z}(g(x)(b)) \leqslant w(a)+w(b),
$$

where the last inequality follows from:

$$
w_{Z}(g(a)(y)) \leqslant \sup _{y^{\prime}} w_{Z}\left(g(a)\left(y^{\prime}\right)\right)=W(g(a)) \leqslant w(a), w_{Z}(g(x)(b)) \leqslant w(b) .
$$

\subsection{Homotopy for weighted categories}

This elementary theory is based on the directed interval $\mathbf{2}=\{0 \rightarrow 1\}$, an order category on two objects, where the only non-trivial arrow has weight $w(0 \rightarrow 1)=1$. The obvious faces $\partial^{ \pm}: \mathbf{1} \rightarrow \mathbf{2}$ are defined on the singleton category $\mathbf{1}=\{*\}$.

A point $x: \mathbf{1} \rightarrow X$ of a small weighted category $X$ is an object of the latter; we will also write $x \in X$. An extended path $a: \mathbf{2} \rightarrow X$ from $x$ to $x^{\prime}$ corresponds to a feasible arrow $a: x \rightarrow x^{\prime}$ of $X$, with a finite weight $w(a)$; in fact, the latter coincides with the Lipschitz weight $\|a\|$, as a functor on $\mathbf{2}$; concatenation of extended paths corresponds to composition in $X$ (strictly associative, with strict identities). Elementary paths correspond to arrows with $w(a) \leqslant 1$, which will also be called 1-morphisms, and cannot generally be concatenated.

The monoidal closed structure described above (Proposition 3.1) gives the elementary cylinder $I X=X \otimes \mathbf{2}$ and its right adjoint, the elementary path $P Y=Y^{\mathbf{2}}$, which consists of the category of 1-morphisms of $Y$ and their arbitrary commutative squares, with weight $W\left(b, b^{\prime}\right)=w(b) \vee w\left(b^{\prime}\right)$. This shows that an elementary homotopy, or elementary natural transformation, $\varphi: f \rightarrow g: X \rightarrow Y$ is the same as a natural transformation between 1-Lipschitz functors, satisfying $w(\varphi(x)) \leqslant 1$ for all $x \in X$. Such homotopies cannot be concatenated, since their vertical composition is no longer elementary, in general. An elementary isomorphism of 1-Lipschitz 
functors will be an elementary natural transformation having an inverse in the same domain; plainly, this corresponds to an invertible natural transformation such that $w(\varphi(x)) \vee w\left(\varphi^{-1}(x)\right) \leqslant 1$, for all points $x$.

Let us consider now an arbitrary functor $\varphi:|X \otimes \mathbf{2}| \rightarrow|Y|$ between the underlying categories, and define its reduced weight $|\varphi|$ as follows (writing $\varphi(x)=\varphi(x, 0 \rightarrow 1$ ), for $x \in X$ )

$$
|\varphi|=\sup _{x} w_{Y}(\varphi(x))=\min \left\{\lambda \in[0, \infty] \mid \text { for all } x \in X, w_{Y}(\varphi(x)) \leqslant \lambda\right\} .
$$

Note now that $\varphi$ sends a map $(a, \operatorname{id}(0))$ of $X \otimes \mathbf{2}$ to $f(a)$, a map $(a, \operatorname{id}(1))$ to $g(a)$ and a map $\left(a: x \rightarrow x^{\prime}, 0 \rightarrow 1\right)$ to

$$
\begin{aligned}
& b=\varphi(a, 0 \rightarrow 1)=g(a) \circ \varphi(x)=\varphi\left(x^{\prime}\right) \circ f(a), \\
& w(b) \leqslant w(f(a))+w\left(\varphi\left(x^{\prime}\right)\right) \leqslant\|f\| \cdot w(a)+|\varphi| \leqslant(\|f\| \vee|\varphi|) \cdot(w(a)+1), \\
& w(b) \leqslant w(g(a))+w(\varphi(x)) \leqslant\|g\| \cdot w(a)+|\varphi| \leqslant(\|g\| \vee|\varphi|) \cdot(w(a)+1) .
\end{aligned}
$$

Therefore, the Lipschitz weight of $\varphi$ as a functor $\varphi:|X \otimes \mathbf{2}| \rightarrow|Y|$, or global weight, is

$$
\|\varphi\|=\|f\| \vee\|g\| \vee|\varphi| \text {. }
$$

A Lipschitz homotopy, or Lipschitz natural transformation, $\varphi: f \rightarrow g: X \rightarrow Y$ will be a natural transformation with a finite global weight $\|\varphi\|$, or equivalently a natural transformation of Lipschitz functors with a finite reduced weight $|\varphi|$. We will write:

(i) $\mathrm{w}_{1}$ Cat, the h-category of weighted categories, with 1-Lipschitz functors and elementary natural transformations,

(ii) $\mathrm{w}_{\infty}$ Cat, the 2-category of weighted categories, with Lipschitz functors and Lipschitz natural transformations.

\subsection{The fundamental weighted category}

Let us come back to a $\delta$-metric space $X$, and construct its fundamental weighted category, working with extended paths modulo extended 2-homotopy.

More precisely, an extended double path in $X$ is a $\mathrm{w}_{\infty}$-map $A: \delta \mathbf{I}^{2} \rightarrow X$. It is an instance of a double homotopy (Subsection 2.5), defined on the point, and the previous results for double homotopies apply; its four faces are paths in $X$, between four vertices. A 2-path is a double path whose faces $\partial_{1}^{\alpha}$ are degenerate; it is a 2homotopy $A: a \prec_{2} b: x \rightarrow x^{\prime}$ between its faces $\partial_{2}^{\alpha}$, which have the same endpoints. A 2-homotopy class of paths $[a]$ is a class of the equivalence relation $\simeq_{2}$ spanned by the preorder $\prec_{2}$.

The fundamental weighted category $\mathrm{w}_{1}(X)$ of the $\delta$-metric space $X$ has for objects the points of $X$; for arrows $[a]: x \rightarrow x^{\prime}$ the 2-homotopy classes of paths from $x$ to $x^{\prime}$, as defined above. Composition - written additively - is induced by concatenation of consecutive paths, and identities come from degenerate paths

$$
[a]+[b]=[a+b], \quad 0_{x}=[e(x)]=\left[0_{x}\right] .
$$

The weight is defined as usual in a quotient, starting from the length of extended 
paths (Definition and Proposition 1.2)

$$
w(\xi)=\inf _{a \in \xi} L(a) .
$$

This is indeed a category, with the same proof as for d-spaces ([9], Theorem 3.2a), based on the constructions of 2-dimensional homotopies in Subsection 2.5a-c. Moreover $\mathrm{w}_{1}(X)$ is (sub)additively weighted, as it follows immediately from the properties of $L$ (Definition and Proposition 1.2)

$$
w\left(0_{x}\right)=0, \quad w([a]+[b]) \leqslant w[a]+w[b] .
$$

On a $\delta_{\infty}$-map $f: X \rightarrow Y$, we get a $\mathrm{w}_{\infty}$-functor $\mathrm{w}_{1}(f): \mathrm{w} \Pi_{1}(X) \rightarrow \mathrm{w}_{1}(Y)$

$$
\begin{array}{ll}
\mathrm{w} \Pi_{1}(f)(x)=f(x), & \mathrm{w} \Pi_{1}(f)[a]=f_{*}[a]=[f a], \\
w\left(f_{*}[a]\right) \leqslant\|f\| . w[a], & \left\|f_{*}\right\| \leqslant\|f\| .
\end{array}
$$

All this forms a functor $\mathrm{w} \Pi_{1}: \delta_{\infty} \mathbf{M t r} \rightarrow \mathrm{w}_{\infty}$ Cat, with values in the category of (small) additively weighted categories and Lipschitz functors; a functor which restricts to $\delta \mathbf{M t r} \rightarrow$ wCat, because of the last inequality above. In particular, $w \Pi_{1}$ preserves Lipschitz isomorphisms and isometric isomorphisms.

Finally, a $\delta_{\infty}$-homotopy $\varphi: f \rightarrow g: X \rightarrow Y$ yields a Lipschitz natural transformation

$$
\begin{aligned}
& \varphi_{*}: f_{*} \rightarrow g_{*}: \mathrm{w} \Pi_{1}(X) \rightarrow \mathrm{w} \Pi_{1}(Y), \\
& w\left(\varphi_{*}(x)\right)=w[\varphi(x)] \leqslant\|\varphi\|, \quad\left\|\varphi_{*}\right\| \leqslant\|\varphi\|,
\end{aligned}
$$

so that $\mathrm{w}_{1}: \delta_{\infty} \mathbf{M t r} \rightarrow \mathrm{w}_{\infty}$ Cat is actually a morphism of h-categories, as well as its restriction $\delta_{1} \mathbf{M t r} \rightarrow \mathrm{w}_{1}$ Cat to the 1-Lipschitz case.

The fundamental weighted category of $X$ is linked to the fundamental groupoid of the underlying space $U X$, by the obvious comparison functor

$$
\mathrm{w} \Pi_{1}(X) \rightarrow \Pi_{1}(U X), \quad x \mapsto x, \quad[a] \mapsto[a] .
$$

\subsection{Geodesics}

In a $\delta$-metric space $X$, we say that an extended path $a: x \rightarrow x^{\prime}$ is a homotopic geodesic if it realises the weight of its class, $L(a)=w[a]$, which amounts to saying that $L(a) \leqslant L\left(a^{\prime}\right)$ for all extended paths $a^{\prime} \simeq_{2} a$.

We say that the space $X$ is geodetically simple if every arrow $\xi: x \rightarrow x^{\prime}$ of its fundamental weighted category $\mathrm{w} \Pi_{1}(X)$ has some representative $a$ which realises its weight $L(a)=w(\xi)$; the path $a$ is then a homotopic geodesic.

We say that $X$ is 1-simple if its fundamental category $\mathrm{w} \Pi_{1}(X)$ is a preorder; all hom sets have at most one arrow.

The $\delta$-metric spaces $\delta \mathbf{R}^{n}, \delta \mathbf{R}^{\otimes n}$ are geodetically simple and 1-simple; all their convex subspaces are also (cf. [9], 3.4a). The pierced plane $(! \delta \mathbf{R})^{2} \backslash\{0\}$ is not geodetically simple, nor 1 -simple. The $\delta$-metric circle $\delta \mathbf{S}^{1}$ is geodetically simple and not 1 -simple; the higher spheres are 1 -simple.

It appears from these examples that being geodetically simple is related somehow to completeness of the $\delta$-metric. Actually, a non-complete space, like $\delta] 0,1[\subset \delta \mathbf{R}$ can be geodetically simple, but it is also true that all its extended paths $x \rightarrow x^{\prime}$ (between two given points) stay in the compact subspace $\delta\left[x, x^{\prime}\right]$. 
Theorem 3.2 (Pasting Theorem - 'Seifert-van Kampen' for fundamental weighted categories). Let $X$ be a $\delta$-metric space; $X_{1}, X_{2}$ two subspaces and $X_{0}=X_{1} \cap X_{2}$.

If $X=\operatorname{int}\left(X_{1}\right) \cup \operatorname{int}\left(X_{2}\right)$, the following diagram of weighted categories and contracting functors (induced by inclusions) is a pushout in ${ }_{\mathrm{w}} \mathbf{C a t}$

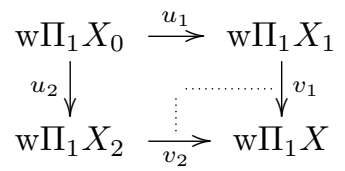

Proof. As in $[\mathbf{9}]$, Theorem 3.6.

\subsection{Homotopy monoids}

The fundamental weighted monoid $w \pi_{1}(X, x)$ of the $\delta$-metric space $X$ at the point $x$ is the (additively) weighted monoid of endoarrows $x \rightarrow x$ in $\mathrm{w}_{1}(X)$. It forms a functor from the (obvious) category $\delta \mathbf{M t r}_{*}$ of pointed $\delta$-metric spaces, to the category of additively weighted monoids ([14], 2.1)

$$
w \pi_{1}: \delta \mathbf{M t r}_{*} \rightarrow \mathrm{w}^{+} \text {Mon, } \quad w \pi_{1}(X, x)=\mathrm{w}_{1}(X)(x, x) .
$$

This functor is strictly homotopy invariant: a pointed homotopy $\varphi: f \rightarrow g:(X, x)$ $\rightarrow(Y, y)$ has, by definition, a trivial path at the base-point $\left(\varphi(x)=0_{y}\right)$, whence the naturality square of every endomap $a: x \rightarrow x$ of $X$ gives $f_{*}[a]=g_{*}[a]$

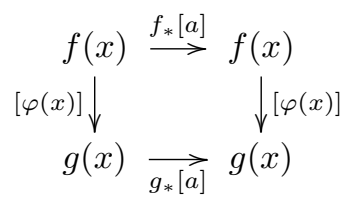

\section{Minimal models}

This is a brief exposition of how the minimal models developed in $[\mathbf{1 3}]$ for the fundamental category of a d-space can be enriched in the present weighted setting. Some knowledge of the main definitions and results of $[\mathbf{1 3}]$ would be useful.

\subsection{The fundamental weighted category of a square annulus}

Let us begin with an elementary example, enriching with a $\delta$-metric the 'square annulus' examined in the Introduction of [13] as an ordered topological space.

Let us start from the $\delta$-metric space $\delta \mathbf{I}^{\otimes 2}$, with

$$
\begin{aligned}
\delta(\mathbf{x}, \mathbf{y}) & =\left(x_{1}-y_{1}\right)+\left(x_{2}-y_{2}\right) & & \text { if } x_{1} \leqslant y_{1}, x_{2} \leqslant y_{2}, \\
& =\infty, & & \text { otherwise, }
\end{aligned}
$$

whose underlying ordered topological space (8) is the ordered topological square $\uparrow[0,1]^{2}$, with euclidean topology and product order. 
Taking out the open square $] 1 / 3,2 / 3\left[^{2}\right.$ (marked with a cross), we get the square annulus $X \subset \delta \mathbf{I}^{\otimes 2}$, with the induced $\delta$-metric

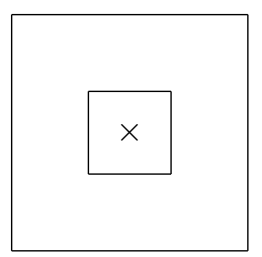

$X$

$X$

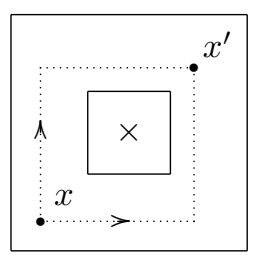

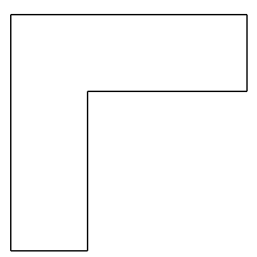

$L$

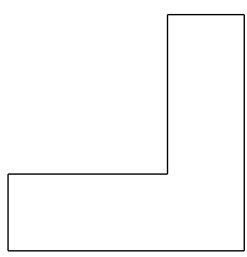

$L^{\prime}$

Its extended paths are thus the Lipschitz order-preserving maps $\delta[0,1] \rightarrow X$ defined on the standard $\delta$-interval, and move 'rightward and upward' (in the weak sense). Extended homotopies of such paths are Lipschitz order-preserving maps $\uparrow[0,1]^{2} \rightarrow X$.

As a consequence of the 'van Kampen' theorem recalled above (using the subspaces $\left.L, L^{\prime}\right)$, the fundamental weighted category $C=\mathrm{w} \Pi_{1}(X)$ is the same as for the underlying ordered topological space; it has some arrow $x \rightarrow x^{\prime}$ provided that $x \leqslant x^{\prime}$ and both points are in $L$ or $L^{\prime}$ (the closed subspaces represented above). Precisely, there are two arrows when $x \leqslant p=(1 / 3,1 / 3)$ and $x^{\prime} \geqslant q=(2 / 3,2 / 3)$ (as in the second figure above), and one otherwise. The weight of an arrow can always be realised as the length of some representative: $X$ is geodetically simple (Subsection 3.4).

Thus, the weighted category $C$ is 'essentially represented' by the full weighted subcategory $E$ on four vertices $0, p, q, 1$ (the central cell does not commute), where each of the four generating arrows has weight $2 / 3$, and the weight of $E$ is linear (strictly additive on composition)
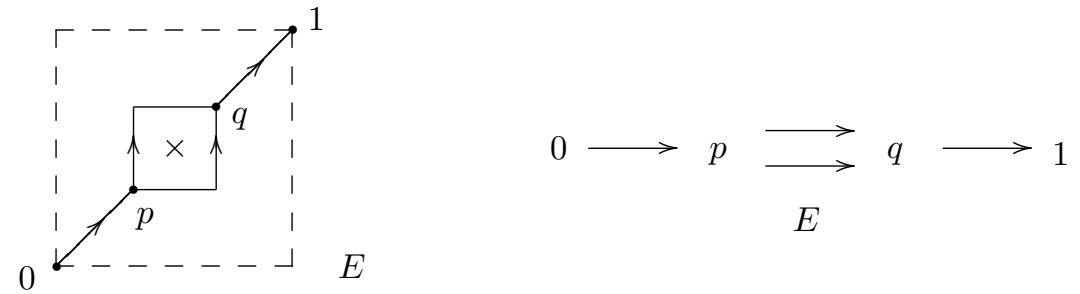

The situation can be analysed as follows, in $E$ :

- the action begins at 0 , from where we move to the point $p$, with weight $2 / 3$,

- $p$ is an (effective) future branching point, where we have to choose between two paths, each of them of weight $2 / 3$, which join at $q$, an (effective) past branching point,

- from where we can only move to 1 , again with weight $2 / 3$, where the process ends. 
(Definitions and properties of regular and branching points can be found in $[\mathbf{1 3}]$, $5.3)$.

Recall that $E$ is not equivalent to $C$, as a category, since $C$ is already a skeleton, in the ordinary sense. In order to make precise how $E$ can 'model' the category $C$, we proved in [13] (and will recall below) that $E$ is both future equivalent and past equivalent to $C$, and is actually the 'join' of a minimal 'future model' with a minimal 'past model' of the latter. All this can now be enriched with weights.

\subsection{Future equivalence of weighted categories}

The notion of future equivalence - a symmetric version of an adjunction, with two units - can be easily transferred from Cat $([\mathbf{1 3}], 2.1)$ to the 2 -category $\mathrm{w}_{\infty} \mathbf{C a t}$, since it makes sense in any 2-category.

Thus, a future equivalence $(f, g ; \varphi, \psi)$ between the weighted categories $C, D$ will consists of a pair of Lipschitz functors and a pair of Lipschitz natural transformations, the units, satisfying two coherence conditions:

$$
\begin{array}{crr}
f: C \rightleftarrows D: g & \varphi: 1_{C} \rightarrow g f, & \psi: 1_{D} \rightarrow f g, \\
f \varphi=\psi f: f \rightarrow f g f, & \varphi g=g \psi: g \rightarrow g f g & \text { (coherence) },
\end{array}
$$

and will be said to be elementary, or 1-Lipschitz, if both functors and both natural transformations are.

Future equivalences compose (as in [13]), and yield an equivalence relation of weighted categories; the elementary ones do not. Dually, past equivalences have counits, in the opposite direction.

In particular, an elementary future retract $i: C_{0} \subset C$ will be a full weighted subcategory having a reflector $p \dashv i$ which is 1-Lipschitz, has a 1-Lipschitz unit $\eta: 1_{C} \rightarrow i p$ and a trivial counit $p i=1$. The coherence conditions of the adjunction $\left(\eta i=1_{i}, p \eta=1_{p}\right)$ show that the fourtuple $(i, p ; 1, \eta)$ is an elementary future equivalence.

A (weighted) pf-presentation (extending [13], 4.2) of the weighted category $C$ will be a diagram consisting of an elementary past retract $P$ and an elementary future retract $F$ of $C$ (which are thus a full coreflective and a full reflective weighted subcategory, respectively) with elementary adjunctions $i^{-} \dashv p^{-}$and $p^{+} \dashv i^{+}$

$$
\begin{array}{ll}
P \underset{p^{-}}{\stackrel{i^{-}}{\gtrless}} C \underset{i^{+}}{\stackrel{p^{+}}{\gtrless}} F & \\
& \\
& \left(p^{-} i^{-}=1, p^{-} \epsilon=1, \epsilon i^{-}=1\right), \\
& \left(p^{+} i^{+}=1, p^{+} \eta=1, \eta i^{+}=1\right) .
\end{array}
$$

\subsection{Spectra}

Coming back to the square annulus $X$ (Subsection 4.1), the weighted category $C=\mathrm{w}_{1}(X)$ has a least full reflective weighted subcategory $F$, which is future equivalent to $C$ and minimal as such. Its objects form the future spectrum $s p^{+}(C)=$ $\{p, 1\}[\mathbf{1 3}]$; also the full weighted subcategory $F=S p^{+}(C)$ on these objects is called 
a future (weighted) spectrum of $C$
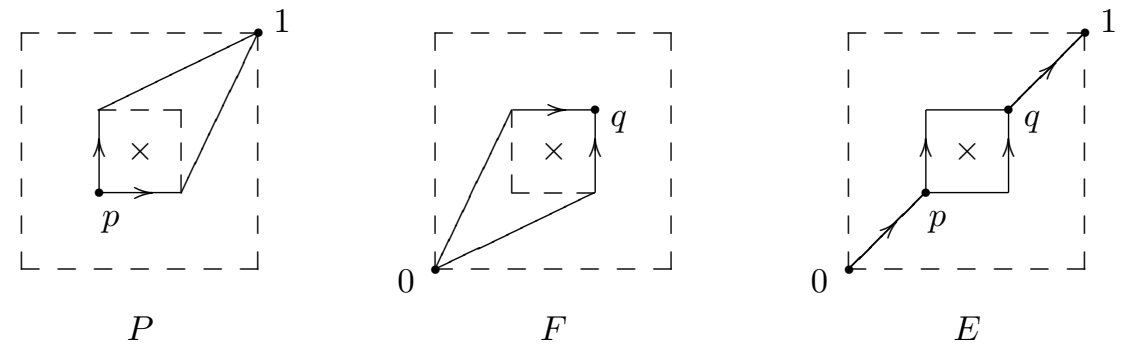

Dually, we have the least full coreflective weighted subcategory $P=S p^{-}(C)$, on the past spectrum $s p^{-}(C)=\{0, q\}$.

Together, they form a (weighted) pf-presentation of $C(12)$, called the spectral pfpresentation. Moreover the (weighted) pf-spectrum $E=S p(C)$ is the full weighted subcategory of $C$ on the set of objects $s p(C)=s p^{-}(C) \cup s p^{+}(C)([\mathbf{1 3}], 7.6)$. $E$ is a strongly minimal injective model of the category $C$ ([13], Theorem 8.4).

\section{Spaces with weighted paths}

We introduce a second setting for weighted algebraic topology, which is more complicated than $\delta$-metric spaces but has finer quotients, as we shall see in the last section. The links between the two settings are developed in Section 6 .

\subsection{Main definitions}

A w-space $X$, or space with weighted paths, will be a topological space together with a weight function $w: X^{\mathbf{I}} \rightarrow[0, \infty]$, or cost function (also written $w_{X}$ ) defined on the set of its (continuous) paths, and satisfying three axioms concerning the constant paths $0_{x}$, the path-concatenation $a+b$ of consecutive paths and strictly increasing reparametrisation (by a strictly increasing continuous map $\rho: \mathbf{I} \rightarrow \mathbf{I}$ ):

(wsp.0) $\quad w\left(0_{x}\right)=0$,

(wsp.1) $\quad w(a+b) \leqslant w(a)+w(b)$,

(wsp.2) $\quad w(a \rho) \leqslant w(a)$, for all points $x$ of $X$, for all pairs of consecutive paths $a, b$. for all paths $a$ and all $\rho: \mathbf{I} \rightarrow \mathbf{I}$ as above.

It is easy to see that the last condition, in the presence of the others, is equivalent to requiring $w(a \rho) \leqslant w(a)$ for all paths $a$ and all increasing continuous maps $\rho: \mathbf{I} \rightarrow$ I which are constant on a finite number of subintervals. It is also equivalent to the conjunction of the following two conditions:

$$
\begin{aligned}
& \left(\text { wsp. } 1^{\prime}\right) \quad w(a) \vee w(b) \leqslant w(a+b), \quad \text { for consecutive paths } a, b \text {. } \\
& \left(\text { wsp. } 2^{\prime}\right) \quad w(a \rho)=w(a), \quad \text { for an increasing homeomorphism } \rho: \mathbf{I} \rightarrow \mathbf{I} \text {. }
\end{aligned}
$$

We shall say that a path is free, feasible or unfeasible when, respectively, its cost is 0 , finite or $\infty$. A w-space will be said to be linear, or strictly additive, if $w(a+b)=w(a)+w(b)$; see Subsection 5.4 for examples. We shall see in Section 6 that linear w-spaces form a coreflective subcategory. Note that we are not asking 
that the weight function be continuous with respect to the compact-open topology of $X^{\mathbf{I}}$; in most examples, this will only be true if we restrict $w$ to the feasible paths (or - equivalently - if we topologise $[0, \infty]$ letting $\infty$ be everywhere dense, which might be interesting).

If $X, Y$ are w-spaces, a $w$-map $f: X \rightarrow Y$, or map of $w$-spaces, or 1-Lipschitz map will be a continuous mapping which decreases costs: $w(f \circ a) \leqslant w(a)$, for all (continuous) paths $a$ of $X$. More generally, a Lipschitz map, or $w_{\infty}$-map $f: X \rightarrow Y$ is a continuous mapping which admits a finite Lipschitz constant $\lambda \in[0, \infty]$, in the sense that $w(f \circ a) \leqslant \lambda . w(a)$, for all continuous paths $a$ in $X$.

We have thus the category wTop of w-spaces and w-maps, embedded in the category $\mathrm{w}_{\infty}$ Top of $\mathrm{w}$-spaces and $\mathrm{w}_{\infty}$-maps. Again, we distinguish between isometric isomorphisms (of wTop) and Lipschitz isomorphisms (of $\mathrm{w}_{\infty}$ Top).

The forgetful functor $U:$ wTop $\rightarrow$ Top has left and right adjoints $D \dashv U \dashv C$, where the discrete weight of $D X$ is the highest possible one, with $w(a)=0$ on the constant paths and $\infty$ on all the others, while the natural, codiscrete weight of $C X$ is the lowest possible one, where all paths have a null cost. Unless otherwise stated, when viewing a topological space as a weighted one we will use the embedding $C:$ Top $\rightarrow$ wTop, where all paths are free.

Note now that the weight function of the w-space $X$ acts on the continuous mappings $a: \mathbf{I} \rightarrow U X$, with values in the underlying topological space; we shall go on writing down, pedantically, such occurrences of $U$. (Viewing these paths as wmaps $D \mathbf{I} \rightarrow X$ would also be correct, but confusing.) Also here, reversing paths by the involution $r: \mathbf{I} \rightarrow \mathbf{I}, r(t)=1-t$, gives the reflected, or opposite, w-space, forming a (covariant) involutive endofunctor

$$
R: \text { wTop } \rightarrow \text { wTop, } \quad R(X)=X^{\mathrm{op}}, \quad w^{\mathrm{op}}(a)=w(a r) .
$$

A w-space is symmetric if it is invariant under reflection. It is reflexive, or selfdual, if it is isometrically isomorphic to its reflection (cf. Subsection 5.4). The notation $X \leqslant X^{\prime}$ will mean that these w-spaces have the same underlying topological space and $w_{X} \leqslant w_{X^{\prime}}$; equivalently, the identity of the underlying space is a w-map $X^{\prime} \rightarrow X$.

\subsection{The weight of a map}

A continuous mapping $f: U X \rightarrow U Y$ between w-spaces takes paths of $U X$ into paths of $U Y$ and thus inherits two weights from the category of weighted sets, the additive weight $([\mathbf{1 4}], 1.5 .3)$

$$
\begin{aligned}
|f|_{0} & =\sup _{a}(w(f \circ a)-w(a)) \\
& =\min \{\lambda \in[0, \infty] \mid \text { for all } a, w(f \circ a) \leqslant \lambda+w(a)\} \quad(a \in \operatorname{Top}(\mathbf{I}, U X)),
\end{aligned}
$$

and the multiplicative weight, or Lipschitz weight ([14], 1.6.3)

$$
\|f\|=|f|_{1}=\sup _{a}(w(f \circ a) / w(a))=\min \{\lambda \mid \text { for all paths } a, w(f \circ a) \leqslant \lambda . w(a)\} .
$$

The first distinguishes w-maps with the condition $|f|_{0}=0$. But here we shall only use the second, written as $\|f\|$, which distinguishes w-maps with the condition $\|f\| \leqslant 1$, and $\mathrm{w}_{\infty}$-maps with the condition $\|f\|<\infty$. With this weight, wTop and 
$\mathrm{w}_{\infty}$ Top are multiplicatively weighted categories [14]; all identities have $\left\|1_{X}\right\| \leqslant 1$ and composition gives $\|g f\| \leqslant\|f\| .\|g\|$.

If $X$ is a w-space and $\lambda \in[0, \infty[$, we write $\lambda X$ the same topological space equipped with the weight $\lambda . w_{X} . \mathrm{A}_{\infty}$-map $f: X \rightarrow Y$ with $\|f\| \leqslant \lambda$ is the same as a w-map $\lambda X \rightarrow Y$.

\subsection{Limits}

The category wTop has all limits and colimits, computed as in Top and equipped with a suitable w-structure.

Thus, for a product $\prod X_{i}$, a path $a: \mathbf{I} \rightarrow U\left(\Pi X_{i}\right)$ of components $a_{i}: \mathbf{I} \rightarrow U X_{i}$ has weight $w\left(a_{i}\right)=\sup w\left(a_{i}\right)$. For a sum $\sum X_{i}$, a path $a: \mathbf{I} \rightarrow U\left(\sum X_{i}\right)$ lives in one component $U X_{i}$ and inherits the weight from the latter.

Given a pair of parallel w-maps $f, g: X \rightarrow Y$, the equaliser is the topological one, with the restricted weight function. The coequaliser is the topological coequaliser $Y / R$, with the induced weight

$$
w(c)=\inf \left(\sum w_{Y}\left(b_{i}\right)\right) \quad(c: \mathbf{I} \rightarrow(U Y) / R),
$$

the inf being taken on all finite families $\left(b_{1}, \ldots, b_{n}\right)$ of paths in $U Y$ such that their projections on the quotient, $p b_{i}: \mathbf{I} \rightarrow(U Y) / R$, are consecutive, and give $c=$ $\left(\left(p b_{1}\right)+\cdots+\left(p b_{n}\right)\right) \rho$ (by $n$-ary concatenation and reparametrisation with an increasing homeomorphism $\mathbf{I} \rightarrow \mathbf{I})$. Of course, if there are no such families, $w(c)=$ $\inf (\emptyset)=\infty$.

The only non-trivial points are verifying that this weight-function satisfies the axioms (wsp.1, $1^{\prime}$ ) of Subsection 5.1. Take $c=c^{\prime}+c^{\prime \prime}: \mathbf{I} \rightarrow Y / R$. First, every pair of decompositions of $c^{\prime}, c^{\prime \prime}$

$$
c^{\prime}=\left(p b_{1}^{\prime}+\cdots+p b_{m}^{\prime}\right) \rho^{\prime}, \quad \quad c^{\prime \prime}=\left(p b_{1}^{\prime \prime}+\cdots+p b_{n}^{\prime \prime}\right) \rho^{\prime \prime},
$$

gives a decomposition $c=\left(p b_{1}^{\prime}+\cdots+p b_{n}^{\prime \prime}\right) \rho$; therefore

$$
\left(w_{Y}\left(b_{1}^{\prime}\right)+\cdots+w_{Y}\left(b_{m}^{\prime}\right)\right)+\left(w_{Y}\left(b_{1}^{\prime \prime}\right)+\cdots+w_{Y}\left(p b_{n}^{\prime \prime}\right)\right) \geqslant w(c),
$$

and $w\left(c^{\prime}\right)+w\left(c^{\prime \prime}\right) \geqslant w(c)$. Second, given a decomposition $c=\left(p b_{1}+\cdots+p b_{n}\right) \rho$, we can always assume that $n=2 k$ (possibly inserting a constant path, without modifying $\left.\sum w_{Y}\left(b_{i}\right)\right)$. Then

$$
\begin{aligned}
& \left(p b_{1}+\cdots+p b_{k}\right)+\left(p b_{k+1}+\cdots+p b_{2 k}\right)=c \rho^{-1}=\left(c^{\prime} \rho^{\prime}\right)+\left(c^{\prime \prime} \rho^{\prime \prime}\right), \\
& c^{\prime} \rho^{\prime}=p b_{1}+\cdots+p b_{k}, \\
& w\left(c^{\prime}\right) \leqslant w_{Y}\left(b_{1}\right)+\cdots+w_{Y}\left(b_{k}\right) \leqslant w_{Y}\left(b_{1}\right)+\cdots+w_{Y}\left(b_{2 k}\right)
\end{aligned}
$$

(for suitable reparametrisations $\left.\rho^{\prime}, \rho^{\prime \prime}\right)$. It follows that $w\left(c^{\prime}\right) \leqslant w(c)$, and similarly for $c^{\prime \prime}$.

Linear w-spaces are not closed under (even binary) products, as we see below. But they are closed under subspaces (plainly), all colimits (by adjointness) and tensor product (Subsection 5.5).

The category $\mathrm{w}_{\infty}$ Top has finite limits and colimits, which can be constructed as above. Even if, now, they are only determined up to Lipschitz isomorphism, we shall keep the previous constructions as privileged ones. Thus, when we write $X \times Y$ in 
$\mathrm{w}_{\infty}$ Top, we still mean that its weight is the $l_{1}$-weight, with $w(a, b)=w(a)+w(b)$; isomorphic constructions will have different names (cf. Subsection 5.5). It is easy to verify that: $\|f \times g\| \leqslant\|f\| \vee\|g\|$.

\subsection{Standard models}

The standard weighted real line, or $w$-line $\mathrm{w} \mathbf{R}$, is the euclidean line with the following weight on all paths $a: \mathbf{I} \rightarrow \mathbf{R}$, equivalently defined by its span or length in $\delta \mathbf{R}(1.2)$ :

$$
w(a)=s p(a)=L(a)
$$

thus, $w(a)$ is finite if and only if $a$ is a (weakly) increasing path, and then $w(a)=$ $a(1)-a(0)$. (General links between $\delta$-metric spaces and w-spaces will be studied in the next section.)

Its cartesian power in wTop, the $n$-dimensional standard $w$-space $\mathrm{w}^{n}$ has $w(a)=\sup _{i}\left(a_{i}(1)-a_{i}(0)\right)$ for all increasing paths $a: \mathbf{I} \rightarrow \mathbf{R}^{n}$ (with respect to the product order, $x \leqslant x^{\prime}$ if and only if $x_{i} \leqslant x_{i}^{\prime}$, for all $i$ ). Plainly, $\mathrm{w} \mathbf{R}$ is linear while the higher dimensional ${ }_{w} \mathbf{R}^{n}$ are not.

The standard $w$-interval $\mathrm{w} \mathbf{I}$ has the subspace structure of the w-line; the standard $w$-cube $\mathrm{w} \mathbf{I}^{n}$ is its $n$-th power, and a subspace of $\mathrm{w}^{n}$. These w-spaces are not symmetric (for $n>0$ ), but reflexive; in particular, the canonical reflecting isomorphism

$$
r: \mathrm{w} \mathbf{I} \rightarrow(\mathrm{w} \mathbf{I})^{\mathrm{op}}, \quad t \mapsto 1-t,
$$

will be used to reflect paths and homotopies. The standard weighted circle ${ }_{\mathrm{w}} \mathbf{S}^{1}$ will be the coequaliser in wTop of the following two pairs of maps (equivalently)

$$
\begin{array}{cc}
\partial^{-}, \partial^{+}:\{*\} \rightrightarrows \mathrm{w} \mathbf{I}, & \partial^{-}(*)=0, \quad \partial^{+}(*)=1, \\
\mathrm{id}, f: \mathrm{w} \mathbf{R} \rightrightarrows \mathrm{w} \mathbf{R}, & f(x)=x+1 .
\end{array}
$$

Thus, the 'standard realisation' of the first coequaliser is the quotient $(\mathrm{w} \mathbf{I}) / \partial \mathbf{I}$, which identifies the endpoints; a feasible path goes around the circle in a specific direction, and its weight measures the length of the path with respect to the length of the circle: $w(a)=L(a)$ in $\delta \mathbf{S}^{1}$. The Lipschitz-isomorphic structure $2 \pi \cdot \mathrm{w} \mathbf{S}^{1}$ is also of interest. Both are linear.

More generally, the weighted n-dimensional sphere will be the quotient of the weighted cube w $\mathbf{I}^{n}$ modulo its (ordinary) boundary $\partial \mathbf{I}^{n}$, while ${ }_{\mathrm{w}} \mathbf{S}^{0}$ has the discrete topology and the unique w-structure

$$
{ }_{\mathrm{w}} \mathbf{S}^{n}=\left(\mathrm{w} \mathbf{I}^{n}\right) /\left(\partial \mathbf{I}^{n}\right) \quad(n>0), \quad \quad \mathrm{w}^{0}=\mathbf{S}^{0}=\{-1,1\} .
$$

All directed spheres are reflexive. Again, ${ }_{w} \mathbf{S}^{1}$ is linear while the higher spheres are not.

\subsection{Tensor product}

The tensor product $X \otimes Y$ of two w-spaces (similar to the tensor product in $\mathbf{w}^{+}$) will be the cartesian product of the underlying topological spaces, with an $l_{1}$-type 
weight (instead of the $l_{\infty}$-one, pertaining to the cartesian product)

$$
w_{\otimes}(a, b)=w_{X}(a)+w_{Y}(b),
$$

where $(a, b): \mathbf{I} \rightarrow U X \times U Y$ denotes the path of components $a: \mathbf{I} \rightarrow U X, b: \mathbf{I} \rightarrow$ $U Y$. Plainly, this defines a symmetric monoidal structure on wTop, with identity the singleton $\mathrm{w}$-space $\{*\}$.

Linear w-spaces are closed under tensor product. In particular, all tensor powers $(\mathrm{w} \mathbf{R})^{\otimes n},(\mathrm{w} \mathbf{I})^{\otimes n}$ and $\left({ }_{\mathrm{w}} \mathbf{S}^{1}\right)^{\otimes n}$ are linear. The following theorem shows that all of them are exponentiable with respect to the tensor product; in particular, the tensor power $(w \mathbf{I})^{\otimes n}$, which is what is relevant for homotopy.

This tensor product extends to $\mathrm{w}_{\infty}$ Top, with $\|f \otimes g\| \leqslant\|f\| \vee\|g\|$. Even if here the tensor product is isomorphic to the cartesian one, we will keep these two constructs distinct.

Theorem 5.1 (Exponentiable w-spaces). Let $Y$ be a linear w-space with a locally compact Hausdorff topology. Then $Y$ is $\otimes$-exponentiable in wTop; for every wspace $Z$, the internal hom

$$
Z^{Y}=\mathrm{w} \boldsymbol{T o p}(Y, Z) \subset \boldsymbol{T} \boldsymbol{T o p}(U Y, U Z),
$$

is the set of w-maps, with the compact-open topology restricted from $(U Z)^{U Y}$ and the following w-structure. A path $c: \mathbf{I} \rightarrow U\left(Z^{Y}\right) \subset(U Z)^{U Y}$ has weight

$$
W(c)=\sup _{b}\left(w_{Z}(e v \circ(c, b))-w_{Y}(b)\right) \quad(\text { for } b: \mathbf{I} \rightarrow U Y)
$$

where $\lambda-\mu$ is the truncated difference in $\mathbf{w}^{+}$. The evaluation mapping

$$
\text { ev }: Z^{Y} \otimes Y \rightarrow Z
$$

is the restriction of the topological one; it is a w-map, the counit of the adjunction.

Proof. (Note. The same proof, conveniently simplified, shows that $\delta \mathbf{M t r}$ is monoidal closed.)

We defer to the end the technical part showing that $W$ is indeed a w-structure. First, the evaluation mapping (14) satisfies the inequality $w_{\otimes}(c, b) \geqslant w_{Z}(e v \circ(c, b))$, because of the adjunction in $\mathbf{w}^{+}$:

$$
\begin{aligned}
& W(c) \geqslant w_{Z}(e v \circ(c, b))-w_{Y}(b) \quad(\text { for all } b: \mathbf{I} \rightarrow U Y), \\
& w_{\otimes}(c, b)=W(c)+w_{Y}(b) \geqslant w_{Z}(e v \circ(c, b)) .
\end{aligned}
$$

Second, the pair $\left(Z^{Y}\right.$, ev: $\left.Z^{Y} \otimes Y \rightarrow Z\right)$ is a universal arrow from the functor $-\otimes Y$ to the object $Z$; given a w-space $X$ and a w-map $f: X \otimes Y \rightarrow Z$, we have to prove that there is precisely one w-map $g: X \rightarrow Z^{Y}$ such that $f$ factors as

$$
e v \circ(g \otimes Y): X \otimes Y \rightarrow Z^{Y} \otimes Y \rightarrow Z .
$$

Indeed, since $Y$ is exponentiable in Top, there exists precisely one continuous mapping $g: U X \rightarrow(U Z)^{U Y}$ such that $f=e v \circ(g \times U Y)$, and it will be sufficient to prove the following two facts.

(a) $\operatorname{Im}(g) \subset Z^{Y}$. For $x \in X$, we must prove that $g(x): Y \rightarrow Z$ is a w-map. And indeed, for every path $b: \mathbf{I} \rightarrow U Y$

$$
w(g(x) \circ b)=w\left(f \circ\left(0_{x}, b\right) \leqslant w_{\otimes}\left(0_{x}, b\right)=w_{X}\left(0_{x}\right)+w_{Y}(b)=w_{Y}(b) .\right.
$$


Homology, Homotopy and Applications, vol. 9(1), 2007

(b) The mapping $g$ is a w-map $X \rightarrow Z^{Y}$. And indeed, for every path $a: \mathbf{I} \rightarrow U A$

$$
\begin{aligned}
W(g \circ a) & =\sup _{b}\left(w_{Z}(e v \circ(g a, b))-w_{Y}(b)\right)=\sup _{b}\left(w_{Z}(f \circ(a, b))-w_{Y}(b)\right) \\
& \leqslant \sup _{b}\left(w_{\otimes}(a, b)-w_{Y}(b)\right)=w_{X}(a) .
\end{aligned}
$$

Finally, we verify the axioms for the hom-weight $W$. First, the constant path $0_{h}: \mathbf{I} \rightarrow Z^{Y}$ at some w-map $h: Y \rightarrow Z$ gives

$$
W\left(0_{h}\right)=\sup _{b}\left(w_{Z}\left(e v \circ\left(0_{h}, b\right)\right)-w_{Y}(b)\right)=\sup _{b}\left(w_{Z}(h b)-w_{Y}(b)\right)=0 .
$$

Second, to prove (wsp.1), let $c=c^{\prime}+c^{\prime \prime}$ be a concatenation of paths in $U\left(Z^{Y}\right)$. We can always rewrite a path $b: \mathbf{I} \rightarrow U Y$ as the concatenation $b=b^{\prime}+b^{\prime \prime}$ of its two halves, so that, using the assumption that $Y$ is linear and letting $\left(b^{\prime}, b^{\prime \prime}\right)$ be an arbitrary pair of consecutive paths in $U Y$,

$$
\begin{aligned}
& W\left(c^{\prime}+c^{\prime \prime}\right)=\sup _{b}\left[w_{Z}\left(e v \circ\left(c^{\prime}+c^{\prime \prime}, b\right)\right)-w_{Y}(b)\right] \\
& \quad=\sup _{b^{\prime} b^{\prime \prime}}\left[w_{Z}\left(e v \circ\left(c^{\prime}+c^{\prime \prime}, b^{\prime}+b^{\prime \prime}\right)\right)-w_{Y}\left(b^{\prime}+b^{\prime \prime}\right)\right] \\
& \quad=\sup _{b^{\prime} b^{\prime \prime}}\left[w_{Z}\left(\left(e v \circ\left(c^{\prime}, b^{\prime}\right)\right)+\left(e v \circ\left(c^{\prime \prime}, b^{\prime \prime}\right)\right)\right)-w_{Y}\left(b^{\prime}\right)-w_{Y}\left(b^{\prime \prime}\right)\right] \\
& \quad \leqslant \sup _{b^{\prime} b^{\prime \prime}}\left[\left(w_{Z}\left(e v \circ\left(c^{\prime}, b^{\prime}\right)\right)-w_{Y}\left(b^{\prime}\right)+w_{Z}\left(e v \circ\left(c^{\prime \prime}, b^{\prime \prime}\right)\right)-w_{Y}\left(b^{\prime \prime}\right)\right]\right. \\
& \quad \leqslant W\left(c^{\prime}\right)+W\left(c^{\prime \prime}\right),
\end{aligned}
$$

since the last term amounts to the previous sup for arbitrary paths $b^{\prime}, b^{\prime \prime}$ in $Y$. (Note that for $\delta$-metric spaces, one would use the fact that every 'path' $\left(y, y^{\prime}\right): \mathbf{2} \rightarrow Y$ can be rewritten as a trivial 'concatenation' $\left(y, y^{\prime}\right)+\left(y^{\prime}, y^{\prime}\right)$, with $d\left(y, y^{\prime}\right)=d\left(y, y^{\prime}\right)+$ $d\left(y^{\prime}, y^{\prime}\right)$.)

Now for (wsp. $\left.1^{\prime}\right)$, we can make our least upper bound smaller by restriction to those paths $b: \mathbf{I} \rightarrow Y$ which are constant on $\left[\frac{1}{2}, 1\right]$, so that $b=b^{\prime}+b^{\prime \prime}$, with an arbitrary $b^{\prime}$ and $b^{\prime \prime}$ constant at the terminal of $b^{\prime}$

$$
\begin{gathered}
W\left(c^{\prime}+c^{\prime \prime}\right) \geqslant \sup _{b^{\prime}}\left(w_{Z}\left(\left(e v \circ\left(c^{\prime}, b^{\prime}\right)\right)+\left(e v \circ\left(c^{\prime \prime}, b^{\prime \prime}\right)\right)\right)-w_{Y}\left(b^{\prime}+b^{\prime \prime}\right)\right) \\
\quad \geqslant \sup _{b^{\prime}}\left(w_{Z}\left(e v \circ\left(c^{\prime}, b^{\prime}\right)\right)-w_{Y}\left(b^{\prime}\right)\right)=W\left(c^{\prime}\right)
\end{gathered}
$$

where, again, we have used the linear property of $Y: w(b)=w\left(b^{\prime}\right)+w\left(b^{\prime \prime}\right)=w\left(b^{\prime}\right)$.

Last, for (wsp. $2^{\prime}$ ), given an increasing homeomorphism $\rho: \mathbf{I} \rightarrow \mathbf{I}$, every path $b^{\prime}$ in $U Y$ can be rewritten as $b \rho$, with $b=b^{\prime} \rho^{-1}$, so that:

$$
\begin{aligned}
& W(c \rho)=\sup _{b}\left(w_{Z}(e v \circ(c \rho, b \rho))-w_{Y}(b \rho)\right)=\sup _{b}\left(w_{Z}(e v \circ(c, b) \circ \rho)-w_{Y}(b \rho)\right) \\
& =W(c) .
\end{aligned}
$$

\subsection{Elementary and extended paths}

Let $X$ be a w-space. An elementary path (resp. an extended path, or Lipschitz path) in $X$ will be a 1-Lipschitz (resp. a Lipschitz) map $a: \mathrm{w} \mathbf{I} \rightarrow X$.

Thus, a continuous mapping $a: \mathbf{I} \rightarrow X$ is an elementary path if and only if $\|a\| \leqslant$ 1 , for the Lipschitz weight $\|a\|$ (13), defined as

$$
\min \{\lambda \in[0, \infty] \mid \text { for all increasing maps } \rho: \mathbf{I} \rightarrow \mathbf{I}, w(a \rho) \leqslant \lambda .(\rho(1)-\rho(0))\}
$$

and is an extended path if and only if $\|a\|<\infty$. Again, elementary paths cannot be concatenated. 
The reflected (elementary or extended) path is obtained in the obvious way

$$
a^{\mathrm{op}}=a r: \mathrm{w} \mathbf{I} \rightarrow X^{\mathrm{op}}, \quad r(t)=1-t .
$$

A reversible extended path is a mapping $a: \mathbf{I} \rightarrow X$ such that both $a$ and $a^{\text {op }}$ are extended paths wI $\rightarrow X$.

In the category wTop, pasting two copies of the standard weighted interval, one after the other, can be realised as $\mathrm{w}[0,2] \subset \mathrm{w} \mathbf{R}$ (or as 2.wI, cf. Subsection 5.2), which is of no help for concatenating paths parametrised on wI, in wTop. But in $\mathrm{w}_{\infty}$ Top this pasting can be realised as wI (Lipschitz-isomorphic to $\mathrm{w}[0,2]$ ) by the standard concatenation pushout

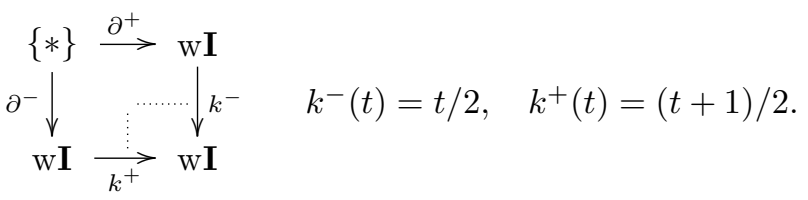

(Again, the diagram above is still in wTop, but is a pushout only in $\mathrm{w}_{\infty}$ Top.) Now, given two consecutive Lipschitz paths $a, b: \mathrm{w} \mathbf{I} \rightarrow X$, with $a(1)=b(0)$, we get a concatenated path

$$
a+b: \mathrm{w} \mathbf{I} \rightarrow X, \quad \quad\|a+b\| \leqslant 2 .(\|a\|+\|b\|),
$$

as follows from the following proposition (or using the pushout 2.wI, in wTop).

We can now treat homotopies as for $\delta$-metric spaces, in Section 2, distinguishing the h-categories $\mathrm{w}_{1}$ Top $\subset$ wTop $\subset \mathrm{w}_{\infty}$ Top, where the intermediated h-category wTop has 1-Lipschitz maps and Lipschitz homotopies. We can also define the fundamental weighted category of a w-space as in Section 3. In particular, concatenation is based on the following result.

Proposition 5.2. For every w-space $X$, the functor $X \times-: \mathrm{w}_{\infty}$ Top $\rightarrow \mathrm{w}_{\infty}$ Top preserves the standard concatenation pushout (15). Moreover, if a map $f: X \times \mathrm{w} \mathbf{I} \rightarrow$ $Y$ comes from the pasting of two 'consecutive' maps $f_{0}, f_{1}: X \times \mathrm{w} \mathbf{I} \rightarrow Y$, we have the following upper bound for its Lipschitz weight:

$$
\|f\| \leqslant 2 .\left(\left\|f_{0}\right\|+\left\|f_{1}\right\|\right) \quad\left(f_{0}=f \circ\left(X \times k^{-}\right), f_{1}=f \circ\left(X \times k^{+}\right)\right) .
$$

Equivalently, one can use the Lipschitz-isomorphic functor $X \otimes-$.

Proof. In Top, the preservation holds because the subspaces $U X \times\left[0, \frac{1}{2}\right]$ and $U X \times$ $\left[\frac{1}{2}, 1\right]$ form a finite closed covering of $U X \times \mathbf{I}$, so that each mapping defined on the latter and continuous on such closed parts is continuous.

Consider then a (topological) map $f: U X \times \mathbf{I} \rightarrow U Y$ coming from the pasting of two maps $f_{0}, f_{1}$ on the topological pushout $U X \times \mathbf{I}$

$$
f(x, t)=f_{0}(x, 2 t), \text { for } 0 \leqslant t \leqslant \frac{1}{2}, \quad f(x, t)=f_{1}(x, 2 t-1), \text { for } \frac{1}{2} \leqslant t \leqslant 1 .
$$

Now let $(a, \rho): \mathbf{I} \rightarrow U X \times \mathbf{I}$ be any feasible path; in particular, $\rho: \mathbf{I} \rightarrow \mathbf{I}$ is an increasing map. If the image of $\rho$ is contained in the first half of $\mathbf{I}$, then $f \circ(a, \rho)=$ 
$f_{0}(a, 2 \rho)$ and

$$
w(f \circ(a, \rho)) \leqslant\left\|f_{0}\right\| \cdot(w(a) \vee 2 w(\rho)) \leqslant 2 .\left\|f_{0}\right\| \cdot w(a, \rho) .
$$

A similar argument holds for the second half. Otherwise, since $\rho$ is increasing, we have $\rho\left(t_{1}\right)=\frac{1}{2}$ at some interior point $\left.t_{1} \in\right] 0,1\left[\right.$; and we can assume that $t_{1}=\frac{1}{2}$ (up to precomposing with an increasing homeomorphism $\sigma: \mathbf{I} \rightarrow \mathbf{I}$, which does not modify the weight of paths, by $\left(\right.$ wsp. $\left.\left.2^{\prime}\right)\right)$. Now the path $f \circ(a, \rho): \mathbf{I} \rightarrow U Y$ is the concatenation of two paths $c_{i}: \mathrm{w} \mathbf{I} \rightarrow U Y$ which factor through the Lipschitz maps $f_{i}$

$$
\begin{aligned}
& c_{0}(t)=f \circ(a(t / 2), \rho(t / 2))=f_{0} \circ(a(t / 2), 2 \rho(t / 2)), \\
& c_{1}(t)=f \circ(a((t+1) / 2), \rho((t+1) / 2))=f_{1} \circ(a((t+1) / 2), 2 \rho((t+1) / 2)-1) .
\end{aligned}
$$

and finally we can conclude that $f$ is Lipschitz, with the upper bound (16)

$$
\begin{aligned}
& w(f \circ(a, \rho)) \leqslant w\left(c_{0}\right)+w\left(c_{1}\right) \leqslant\left(\left\|f_{0}\right\|+\left\|f_{1}\right\|\right) \cdot(w(a) \vee 2 w(\rho)) \\
& \quad \leqslant 2 .\left(\left\|f_{0}\right\|+\left\|f_{1}\right\|\right) \cdot w(a, \rho) .
\end{aligned}
$$

\section{Linear and metrisable w-spaces}

The span and length function of a $\delta$-metric space $X$, defined in Definition and Proposition 1.2, allow us to construct the w-spaces $\mathbf{s p} X$ (Subsection 6.2) and $\mathbf{L} X$; the latter is linear (Subsection 6.3).

\subsection{Linear w-spaces}

First, we want to observe that linear w-spaces form a full subcategory $\mathrm{Lw}_{\infty}$ Top of $\mathrm{w}_{\infty}$ Top, which has a coreflector $L$, right adjoint to the embedding $U$

$$
U: \mathrm{Lw}_{\infty} \text { Top } \rightleftarrows \mathrm{w}_{\infty} \text { Top : } L \quad(U \dashv L) .
$$

In fact, for a w-space $X$, there is a linearised w-space $L(X)$ on the same underlying topological space, endowed with the least linear weight $L \geqslant w$

$$
\begin{array}{lr}
L(a)=\sup _{\mathbf{t}} \sum_{j} w\left(a\left(t_{j-1}, t_{j}\right)\right) & \left(0=t_{0}<t_{1}<\cdots<t_{p}=1\right), \\
a\left(t_{j-1}, t_{j}\right)(t)=a\left((1-t) \cdot t_{j-1}+t . t_{j}\right) & (0 \leqslant t \leqslant 1) .
\end{array}
$$

Note that we have written $a\left(t_{j-1}, t_{j}\right): \mathbf{I} \rightarrow X$, the restriction of the path $a$ to the interval $\left[t_{j-1}, t_{j}\right]$ reparametrised on the standard interval.

Thus $L(X) \geqslant X$, and $L(X)=X$ if and only if the w-space $X$ is linear. These relations form, respectively, the counit $U L \rightarrow 1$ and the unit $L U=1$ of the adjunction.

All this restricts to contractions, giving a full coreflective subcategory LwTop of wTop. Therefore, linear w-spaces are closed under colimits in wTop.

\subsection{Span-metrisable w-spaces}

Now, let us construct an adjunction

$$
\delta: \mathrm{w}_{\infty} \text { Top } \rightleftarrows \delta_{\infty} \text { Mtr : sp } \quad(\delta \dashv \mathrm{sp}) .
$$


First, the functor

$$
\delta: \mathrm{w}_{\infty} \text { Top } \rightarrow \delta_{\infty} \text { Mtr, } \quad\|\delta f\| \leqslant\|f\|, \quad(\delta: \text { wTop } \rightarrow \delta \text { Mtr }),
$$

sends a w-space $X$ to the $\delta$-metric space $\delta X$, consisting of the same set with

$$
\delta\left(x, x^{\prime}\right)=\inf _{a} w(a),
$$

where $a: \delta \mathbf{I} \rightarrow X$ varies in the set of extended paths in $X$, from $x$ to $x^{\prime}$. The $\delta$ metric spaces obtained in this way from a w-space will be said to be geodetic. Plainly, if $f: X \rightarrow X^{\prime}$ is a $\mathrm{w}_{\infty}-\mathrm{map}, \boldsymbol{\delta} f=f: \boldsymbol{\delta} X \rightarrow \boldsymbol{\delta} X^{\prime}$ is continuous and satisfies the inequality of (20), whence it is a $\delta_{\infty}$-map (and 1-Lipschitz if $f$ is).

Second, the functor

$$
\mathbf{s p}: \delta_{\infty} \mathbf{M t r} \rightarrow \mathrm{w}_{\infty} \text { Top, } \quad\|\mathbf{s p} f\| \leqslant\|f\|, \quad(\mathbf{s p}: \delta \mathbf{M t r} \rightarrow \text { wTop}),
$$

has essentially been constructed in Section 1 . For a $\delta$-metric space $Y$, we let $\operatorname{sp} Y$ be the same set equipped with the symmetric topology (Subsection 1.6) and with the weight-function $s p$ defined in (7)

$$
\operatorname{sp}(a)=\sup _{\mathbf{t}} \delta\left(a\left(t_{0}\right), a\left(t_{1}\right)\right) \quad\left(0 \leqslant t_{0}<t_{1} \leqslant 1\right),
$$

which has already been seen to satisfy the axioms of w-spaces (Definition and Proposition 1.2).

The w-spaces obtained in this way will be said to be span-metrisable. On maps, we take again the same underlying mapping.

These two functors form an idempotent adjunction $\delta \dashv \mathbf{s p}$, which restricts to a (covariant) Galois connection whenever we fix the underlying set; in fact, the two functors do not change the latter, while unit and counit reduce to inequalities

$$
X \geqslant \operatorname{sp}(\boldsymbol{\delta} X), \quad \boldsymbol{\delta}(\mathbf{s p} Y) \geqslant Y,
$$

where $X$ is a w-space and $Y$ a $\delta$-metric space. (For idempotent adjunctions, see [1] Section 6 and [17] Lemma 4.3.)

The adjunction gives an equivalence between the full subcategories of

(a) span-metrisable $w$-spaces, characterised by the condition $X=\mathbf{s p}(\boldsymbol{\delta} X)$, or, equivalently by the condition $X=\mathbf{s p} Y$ for a suitable $\delta$-metric structure $Y$ (on the same set),

(b) geodetic $\delta$-metric spaces, characterised by the condition $Y=\boldsymbol{\delta}(\mathbf{s p} Y)$, or, equivalently by the condition $Y=\boldsymbol{\delta} X$ for some weighted structure $X$ on the associated topological space.

Restricting to 1-Lipschitz maps, span-metrisable w-spaces form a reflective subcategory of wTop, closed under limits, while geodetic $\delta$-metric spaces form a coreflective subcategory of $\delta \mathbf{M t r}$, closed under colimits.

In the standard examples of Subsection 5.4, the standard w-line is span-metrisable, ${ }_{\mathrm{w}} \mathbf{R}=\mathbf{s p}(\delta \mathbf{R})$ and the standard $\delta$-line is geodetic, $\boldsymbol{\delta}\left({ }_{\mathrm{w}} \mathbf{R}\right)=\delta \mathbf{R}$. Similarly, in higher dimension, $\mathbf{w}^{n}=\mathbf{s p}\left(\delta \mathbf{R}^{n}\right)$ and $\boldsymbol{\delta}\left(\mathrm{w} \mathbf{R}^{n}\right)=\delta \mathbf{R}^{n}$; this also holds for the standard interval and its powers.

The standard $\delta$-circle $\delta \mathbf{S}^{1}=\boldsymbol{\delta}\left({ }_{\mathrm{w}} \mathbf{S}^{1}\right)$ is geodetic, while the circle $\mathbf{S}^{1}$ with the euclidean metric of $\mathbf{R}^{2}$ is not, since $\boldsymbol{\delta}\left(\mathbf{s p} \mathbf{S}^{1}\right)$ has the obvious geodetic distance, 
which is bigger. The standard $\mathrm{w}$-circle $\mathrm{w}^{1}$ is not span-metrisable, since the weight (i.e. length) of its feasible paths has no finite upper bound, while the $\delta$-metric of $\delta \mathbf{S}^{1}=\boldsymbol{\delta}\left(\mathrm{w} \mathbf{S}^{1}\right)$ cannot exceed 1.

\subsection{The length adjunction.}

The span-adjunction (19) and the adjunction of linear w-spaces (18) give a composed adjunction, idempotent again

$$
\delta: \mathrm{Lw}_{\infty} \text { Top } \rightleftarrows \delta_{\infty} \mathbf{M t r}: \mathbf{L} \quad(\boldsymbol{\delta} \dashv \mathbf{L}) .
$$

Now, $\boldsymbol{\delta}$ is the restriction of the functor (20), and equips a linear w-space $X$ with the geodetic $\delta$-metric $\delta\left(x, x^{\prime}\right)=\inf _{a} w(a)$. On the other hand, $\mathbf{L}=L \circ$ sp takes a $\delta$-metric space $Y$ to the same set equipped with the symmetric topology (Subsection 1.6) and with the (linear) weight-function $L$ which we have already defined in Definition and Proposition 1.2,

$$
L(a)=\sup _{\mathbf{t}} \sum \delta\left(a\left(t_{j-1}\right), a\left(t_{j}\right)\right) \quad\left(0=t_{0}<t_{1}<\cdots<t_{p}=1\right) .
$$

Also, here maps are left 'unchanged' and $\|\boldsymbol{\delta} f\| \leqslant\|f\|,\|\mathbf{L} f\| \leqslant\|f\|$, so that the adjunction restricts to contractions.

\subsection{Length-metrisable w-spaces}

The length adjunction (21) also becomes a (covariant) Galois connection whenever we fix the underlying set; unit and counit reduce to inequalities

$$
X \geqslant \mathbf{L}(\boldsymbol{\delta} X), \quad \boldsymbol{\delta}(\mathbf{L} Y) \geqslant Y,
$$

where $X$ is a linear w-space and $Y$ a $\delta$-metric space.

The adjunction gives thus an equivalence between the full subcategories of:

(a) length-metrisable w-spaces, characterised by the condition $X=\mathbf{L}(\boldsymbol{\delta} X)$, or, equivalently by the condition $X=\mathbf{L} Y$ for some $\delta$-metric structure $Y$ on the same set (all such w-spaces are linear),

(b) linearly geodetic $\delta$-metric spaces, characterised by the condition $Y=\boldsymbol{\delta}(\mathbf{L} Y)$, or $Y=\boldsymbol{\delta} X$ for some linear weight $X$ on the associated topological space.

Thus, a linearly geodetic $\delta$-metric space is geodetic; the converse need not be true. For instance, the $\delta$-metric subspace $Y \subset \delta \mathbf{R}^{2}$ consisting of the union of the axes is geodetic, but not linearly so; the points $y=(-1,0)$ and $y^{\prime}=(0,1)$ have $\delta\left(y, y^{\prime}\right)=1$ but all feasible paths $a$ in $Y$, from $y$ to $y^{\prime}$, have length $L(a)=2$.

The two notions of 'metrisability' of w-spaces are not comparable. Indeed, the ${ }_{\mathrm{w}}$-line ${ }_{\mathrm{w}} \mathbf{R}$ is metrisable in both senses. The $\mathrm{w}$-plane ${ }_{\mathrm{w}} \mathbf{R}^{2}$ is span-metrisable and not linear, hence not length-metrisable. The standard w-circle w $\mathbf{S}^{1}$ (Subsection 5.4) is only length-metrisable. Finally, in Theorem 7.1, we will show that the irrational rotation w-space $W_{\vartheta}$ has a trivial $\delta$-metric on $\delta W_{\vartheta}$ (always zero), whence it is neither span- nor length-metrisable. 


\subsection{Directed spaces}

Finally, we have a forgetful functor

$$
\mathrm{d}: \mathrm{w}_{\infty} \text { Top } \rightarrow \mathrm{d} \text { Top }
$$

which sends a w-space to the same topological space, equipped with the distinguished paths obtained from the feasible ones, by reparametrisation along weakly increasing maps $\mathbf{I} \rightarrow \mathbf{I}$.

Composing $\mathbf{L}: \delta_{\infty} \mathbf{M t r} \rightarrow \mathrm{Lw}_{\infty}$ Top with the latter, we get the forgetful functor $\delta_{\infty} \mathbf{M t r} \rightarrow$ dTop already considered in Subsection 1.7, which distinguishes the L-feasible paths of a $\delta$-metric space - already closed under increasing reparametrisation.

\section{Weighted noncommutative tori and their classification}

The irrational rotation w-space $W_{\vartheta}$ (Subsection 7.1 ) has classifications similar to those of the irrational rotation $\mathrm{C}^{*}$-algebras $A_{\vartheta}$. In [12], analogous results were obtained for 'normed' cubical sets and their 'normed' homology - an earlier occurrence of weighted algebraic topology; cubical sets give weaker results, without the metric aspects (cf. [11]). Throughout this section $\vartheta$ is an irrational number.

\subsection{Irrational rotation $\mathrm{w}$-spaces.}

Let us begin recalling some well-known 'noncommutative spaces'.

First, take the line $\mathbf{R}$ and its (dense) additive subgroup $G_{\vartheta}=\mathbf{Z}+\vartheta \mathbf{Z}$, acting on the former by translations. In Top, the orbit space $\mathbf{R} / G_{\vartheta}$ is trivial: an uncountable set with the coarse topology. In $\delta \mathbf{M t r}$, the quotient $\delta \mathbf{R} / G_{\vartheta}$ is trivial as well: an uncountable set with the null distance.

In noncommutative geometry, this set is 'interpreted' as the (noncommutative) $\mathrm{C}^{*}$-algebra $A_{\vartheta}$, generated by two unitary elements $u, v$ under the relation $v u=$ $\exp (2 \pi i \vartheta) . u v$, and called the irrational rotation algebra associated with $\vartheta$, or also a noncommutative torus $[\mathbf{5}, \mathbf{6}, \mathbf{2 0}, \mathbf{1 9}]$. Both its complex K-theory groups are two-dimensional.

An important achievement of K-theory $[\mathbf{1 9}, \mathbf{2 0}]$ classifies these algebras by proving that $K_{0}\left(A_{\vartheta}\right) \cong \mathbf{Z}+\vartheta \mathbf{Z}$ as an ordered subgroup of $\mathbf{R}$; moreover, the traces of the projections of $A_{\vartheta}$ form the set $G_{\vartheta} \cap[0,1]$. It follows that $A_{\vartheta}$ and $A_{\vartheta}$ are isomorphic if and only if $\vartheta^{\prime} \in \mathbf{Z} \pm \vartheta([\mathbf{2 0}]$, Theorem 2) and strongly Morita equivalent if and only if $\vartheta$ and $\vartheta^{\prime}$ are equivalent modulo the fractional action (on the irrationals) of the group $G L(2, \mathbf{Z})$ of invertible integral $2 \times 2$ matrices ([20], Theorem 4 )

$$
\left(\begin{array}{ll}
a & b \\
c & d
\end{array}\right) \cdot t=(a t+b) /(c t+d) \quad(a, b, c, d \in \mathbf{Z} ; a d-b c= \pm 1)
$$

(or the action of the projective general linear group $P G L(2, \mathbf{Z})$ on the projective line). Since the group $G L(2, \mathbf{Z})$ is generated by the matrices

$$
R=\left(\begin{array}{ll}
0 & 1 \\
1 & 0
\end{array}\right), \quad T=\left(\begin{array}{ll}
1 & 1 \\
0 & 1
\end{array}\right),
$$

the orbit of $\vartheta$ is its closure $\{\vartheta\}_{R T}$ under the transformations $R(t)=t^{-1}$ and $T^{ \pm 1}(t)$ 
$=t \pm 1($ on $\mathbf{R} \backslash \mathbf{Q})$.

We now show how one can obtain similar results with the w-space naturally arising from the action of $G_{\vartheta}$ on the w-line; the point is to replace a topologicallytrivial orbit space $\mathbf{R} / G$ with the corresponding quotient of the standard w-line w $\mathbf{R}$ by a procedure analogous to the one followed in $[\mathbf{1 1}, \mathbf{1 2}]$ for cubical sets or weighted cubical sets.

We are thus led to consider the irrational rotation w-space

$$
W_{\vartheta}=(\mathrm{w} \mathbf{R}) / G_{\vartheta},
$$

whose feasible paths reduce to the projection of the feasible paths of $\mathrm{w} \mathbf{R}$, as we now prove. It will be useful to use the following standard weight on the additive groups $\mathbf{R}$ and $G_{\vartheta}$

$$
w(x)=\delta(0, x),
$$

i.e. $w(x)=x$ when $x \geqslant 0, w(x)=\infty$ otherwise, and the (restricted) standard weight $w(x)=x$ on the additive monoids $\mathbf{R}^{+}$and $G_{\vartheta}^{+}=G_{\vartheta} \cap \mathbf{R}^{+}$formed by the elements of finite weight.

\section{Theorem 7.1.}

(a) The fundamental weighted monoid of $W_{\vartheta}$ at each point $\bar{x} \in \mathbf{R} / G_{\vartheta}$ is isometrically isomorphic to the additive weighted monoid $G_{\vartheta}^{+}$, via the weight function

$$
w: w \pi_{1}\left(W_{\vartheta}, \bar{x}\right) \rightarrow\left[0, \infty\left[, \quad \operatorname{Im}(w)=G_{\vartheta}^{+} .\right.\right.
$$

(b) Choosing a representative $x \in \mathbf{R}$ of $\bar{x}$, for every feasible path $\bar{a}: \mathrm{w} \mathbf{I} \rightarrow W_{\vartheta}$ starting at $\bar{x}$ there is precisely one increasing path $a: \mathrm{w} \mathbf{I} \rightarrow \mathbf{R}$ which lifts it and starts at $x$. Moreover, the weight of $\bar{a}$ in $W_{\vartheta}$ coincides with the weight of $a, w(a)=a(1)-a(0)=a(1)-x$.

(c) The w-space $W_{\vartheta}$ is linear; the associated metric space $\delta W_{\vartheta}$ (20) is codiscrete, with $\delta(\bar{x}, \bar{y})$ always zero, so that $W_{\vartheta}$ is neither span- nor length-metrisable.

Proof. We begin by proving (b). Take a feasible path $\bar{a}$ : $\mathrm{w} \mathbf{I} \rightarrow W_{\vartheta}$ starting at $\bar{x}$, and choose a representative $x \in \mathbf{R}$ of the latter. Then there exists some finite family $a_{1}, \ldots, a_{p}$ of feasible (i.e., increasing) paths in ${ }^{\mathrm{R}} \mathbf{R}$ such that the projections $\bar{a}_{j}$ are consecutive and give $\bar{a}=\bar{a}_{1}+\cdots+\bar{a}_{p}$; further, $w(\bar{a})$ is the greatest lower bound of $\sum w\left(a_{j}\right)$ (for such families).

Now, up to $G_{\vartheta}$-translations, we may assume that $a_{1}$ starts at $x$ and all $a_{j}$ are consecutive (without changing their weight and the concatenation of projections). Thus $a=a_{1}+\cdots+a_{p}$ projects to $\bar{a}$ with $w(a)=\sum w\left(a_{j}\right)$, since $\mathrm{w} \mathbf{R}$ is linear. It follows that $w(\bar{a})$ is the greatest lower bound of $w(a)$, where $a$ varies among the paths in $\mathbf{R}$ which start at $x$ and lift $\bar{a}$.

But there is only one path which satisfies these conditions. Indeed, if $b$ also does, the image of the continuous mapping $a-b: \mathbf{I} \rightarrow \mathbf{R}$ must be contained in $G_{\vartheta}$, which is totally disconnected; thus $a-b$ is constant, and $a(0)=x=b(0)$ gives $a=b$. It follows that $w(\bar{a})=w(a)$, where $a$ is the unique path in $\mathbf{R}$ which starts at $x$ and lifts $\bar{a}$. 
For (c), we have proved above that $W_{\vartheta}$ is linear. The other assertions are obvious, taking into account the characterisations of span- and length-metrisable w-spaces, in Sections 6.2 and 6.4 .

For (a), let us consider the weight function (22). First, we show that its image is $G_{\vartheta}^{+}$. For a loop $\bar{a}$, we have $w(\bar{a})=w(a)$ where the (increasing) lifting $a$ starts at $x$ and ends at some $x^{\prime} \geqslant x$, which also projects to $\bar{x}$; thus $w(\bar{a})=x^{\prime}-x \in G_{\vartheta}^{+}$. On the other hand, if $g \in G_{\vartheta}^{+}$, any increasing path $a: x \rightarrow x+g$ projects to a loop at $\bar{x}$, whose weight is $g$.

Finally, we must prove that the weight function is injective. Let $\bar{a}, \bar{b}$ be two loops at $\bar{x}$ with the same weight $g \in G_{\vartheta}^{+}$, and let $a, b$ be their lifting starting at $x$; again they have the same weight $g$, which means that they end at the same point $x^{\prime}=x+g$. Then the increasing path $c=a \vee b: \mathbf{I} \rightarrow \mathbf{R}$ also goes from $x$ to $x^{\prime}$; since $a \leqslant c$, the affine interpolation from $a$ to $c$ is an extended 2-homotopy $a \prec_{2} c$ (Subsection 3.3); similarly, $b \prec_{2} c$ and $a \simeq_{2} b$, which projects to $[\bar{a}]=[\bar{b}]$.

Theorem 7.2 (Theorem A (Isometric classification)). The w-spaces $\mathrm{w} \mathbf{R} / G_{\vartheta}$ and ${ }_{\mathrm{W}} \mathbf{R} / G_{\vartheta^{\prime}}$ are isometrically isomorphic if and only if $G_{\vartheta}^{+}=G_{\vartheta^{\prime}}^{+}$(as subsets of $\mathbf{R}$ ), if and only if $G_{\vartheta}=G_{\vartheta^{\prime}}$, if and only if $\vartheta^{\prime} \in \mathbf{Z} \pm \vartheta$.

Proof. If our w-spaces are isometrically isomorphic, their fundamental weighted monoids (independently of the base point) are $G_{\vartheta}^{+} \cong G_{\vartheta^{\prime}}^{+}$(isometrically). Since the values of the weight $w: G_{\vartheta}^{+} \rightarrow \mathbf{R}$ form the set $G_{\vartheta}^{+}$, it follows that $G_{\vartheta}^{+}=G_{\vartheta^{\prime}}^{+}$, which implies that $G_{\vartheta}$ (the additive subgroup of $\mathbf{R}$ generated by $G_{\vartheta}^{+}$) coincides with $G_{\vartheta^{\prime}}$. If this is the case, then $\vartheta=a+b \vartheta^{\prime}$ and $\vartheta^{\prime}=c+d \vartheta$ for suitable integers $a, b, c, d$; whence $\vartheta=a+b c+b d \vartheta$ and $d= \pm 1$, so that $\vartheta^{\prime}=c \pm \vartheta$. Finally, if $\vartheta^{\prime} \in \mathbf{Z} \pm \vartheta$, then $G_{\vartheta}=G_{\vartheta^{\prime}}$ and ${ }^{\mathrm{w}} \mathbf{R} / G_{\vartheta}=\mathrm{w} \mathbf{R} / G_{\vartheta^{\prime}}$.

Theorem 7.3 (Theorem B (Lipschitz classification)). The $w$-spaces $\mathrm{w} \mathbf{R} / G_{\vartheta}$ and ${ }_{\mathrm{W}} \mathbf{R} / G_{\vartheta^{\prime}}$ are Lipschitz isomorphic if and only if the equivalent conditions of the following lemma hold.

Proof. One implication follows from Theorem 7.1, namely if our w-spaces are Lipschitz isomorphic, their fundamental weighted monoids $G_{\vartheta}^{+}$and $G_{\vartheta^{\prime}}^{+}$also are, by the functorial properties of $\mathrm{w}_{1}$ (Subsection 3.3). For the converse, let $\vartheta^{\prime}$ belong to the closure $\{\vartheta\}_{R T}$; it suffices to consider the cases $\vartheta^{\prime} \in \vartheta+\mathbf{Z}$ and $\vartheta^{\prime}=\vartheta^{-1}$. In the first case, $G_{\vartheta}$ and $G_{\vartheta^{\prime}}$ coincide, as well as their action on wR; in the second, the Lipschitz isomorphism of w-spaces

$$
f: \mathrm{w} \mathbf{R} \rightarrow \mathrm{w} \mathbf{R}, \quad f(t)=|\vartheta| . t,
$$

restricts to an isomorphism $f^{\prime}: G_{\vartheta} \rightarrow G_{\vartheta^{\prime}}$, obviously consistent with the actions $\left(f(t+g)=f(t)+f^{\prime}(g)\right)$, and induces a Lipschitz isomorphism $\mathrm{w} \mathbf{R} / G_{\vartheta} \rightarrow \mathrm{w} \mathbf{R} / G_{\vartheta^{\prime}}$.

Lemma 7.1. Let $\vartheta, \vartheta^{\prime}$ be irrationals. The following conditions are equivalent:

(a) the weighted groups $G_{\vartheta}$ and $G_{\vartheta^{\prime}}$ are Lipschitz isomorphic,

(b) the weighted monoids $G_{\vartheta}^{+}$and $G_{\vartheta^{\prime}}^{+}$are Lipschitz isomorphic,

(c) $G_{\vartheta}$ and $G_{\vartheta^{\prime}}$ are isomorphic as ordered groups (with respect to the total orders induced by $\mathbf{R}$ ), 
(d) $\vartheta$ and $\vartheta^{\prime}$ are conjugate under the action of $G L(2, \mathbf{Z})$ (Subsection \%.1),

(e) $\vartheta^{\prime}$ belongs to the closure $\{\vartheta\}_{R T}$ of $\{\vartheta\}$ under the mappings $R(t)=t^{-1}$ and $T^{ \pm 1}(t)=t \pm 1$.

Proof. The equivalence of the last three conditions is well-known, within the classification of the $\mathrm{C}^{*}$-algebras $A_{\vartheta}$ up to strong Morita equivalence. It is also proved in $[\mathbf{1 1}]$, Lemma 4.7 .

Further, (a) implies (b), because $G_{\vartheta}^{+}$is the monoid of elements of $G_{\vartheta}$ having a finite weight. And (b) implies (c), because $G_{\vartheta}$ is the group canonically associated to the cancellative monoid $G_{\vartheta}^{+}$, ordered with the latter as a positive cone.

Finally, to prove that (e) implies (a), let $\vartheta^{\prime}$ belong to the closure $\{\vartheta\}_{R T}$; again it suffices to consider the cases $\vartheta^{\prime} \in \vartheta+\mathbf{Z}$ and $\vartheta^{\prime}=\vartheta^{-1}$. In the first, $G_{\vartheta}=G_{\vartheta^{\prime}}$; in

the second, the Lipschitz isomorphism of weighted spaces $f:{ }_{\mathrm{w}} \mathbf{R} \rightarrow{ }_{\mathrm{w}} \mathbf{R}$ considered above (23) restricts to a Lipschitz isomorphism of weighted abelian groups $G_{\vartheta} \rightarrow$ $G_{\vartheta^{\prime}}$.

\section{References}

[1] H. Applegate and M. Tierney, Categories with models, in: Seminar on Triples and Categorical Homology Theory, Lecture Notes in Mathematics 80, Springer, Berlin, 1969, p. 156-244.

[2] R. Betti and M. Galuzzi, Categorie normate, Boll. Un. Mat. Ital. 11 (1975), $66-75$.

[3] N. Bourbaki, Topologie générale, Ch. 10, Hermann, Paris, 1961.

[4] R. Brown, Topology, Ellis Horwood, Chichester, 1988.

[5] A. Connes, C*-algèbres et géométrie différentielle, C.R. Acad. Sci. Paris Sér. A 290 (1980), 599-604.

[6] A. Connes, Noncommutative Geometry, Academic Press, San Diego CA, 1994.

[7] M. Grandis, Cubical homotopical algebra and cochain algebras, Ann. Mat. Pura Appl. 170 (1996), 147-186.

[8] M. Grandis, Categorically algebraic foundations for homotopical algebra, Appl. Categ. Structures 5 (1997), 363-413.

[9] M. Grandis, Directed homotopy theory, I. The fundamental category, Cah. Topol. Géom. Différ. Catég. 44 (2003), 281-316.

[10] M. Grandis, Directed homotopy theory, II. Homotopy constructs, Theory Appl. Categ. 10 (2002), No. 14, 369-391.

[11] M. Grandis, Directed combinatorial homology and noncommutative tori (The breaking of symmetries in algebraic topology), Math. Proc. Cambridge Philos. Soc. 138 (2005), 233-262.

[12] M. Grandis, Normed combinatorial homology and noncommutative tori, Theory Appl. Categ. 13 (2004), No. 7, 114-128.

[13] M. Grandis, The shape of a category up to directed homotopy, Theory Appl. Categ. 15 (2005), No. 4, 95-146. 
[14] M. Grandis, Categories, norms and weights, Dip. Mat. Univ. Genova, Preprint 538 (2006). http://www.dima. unige.it/ grandis/wCat.pdf.

[15] J.C. Kelly, Bitopological spaces, Proc. London Math. Soc. 13 (1963), 71-89.

[16] S. Krishnan, A convenient category of locally ordered spaces, presented at GETCO 2005. Unpublished Manuscript. http://www.math.uchicago.edu/ $\sim$ sanjeevi/

[17] J. Lambek and P.J. Scott, Introduction to Higher Order Categorical Logic, Cambridge University Press, Cambridge, 1986.

[18] F.W. Lawvere, Metric spaces, generalized logic and closed categories, Rend. Sem. Mat. Fis. Univ. Milano 43 (1974), 135-166. Republished in: Repr. Theory Appl. Categ. 1 (2002), 1-37.

[19] M. Pimsner and D. Voiculescu, Imbedding the irrational rotation $\mathrm{C}^{*}$-algebra into an AF-algebra, J. Operator Theory 4 (1980), 93-118.

[20] M.A. Rieffel, C*-algebras associated with irrational rotations, Pacific J. Math. 93 (1981), 415-429.

\author{
Marco Grandis grandis@dima.unige.it \\ Dipartimento di Matematica \\ Università di Genova \\ Via Dodecaneso 35 \\ 16146-Genova, Italy
}

This article is available at http://intlpress.com/HHA/v9/n1/a10 